\title{
MAGNETIC MEASUREMENTS ON THE TCV TOKAMAK
}

J.-M. Moret, F. Bühlmann, D. Fasel, F. Hofmann, G. Tonetti

Submitted for publication in

Review of Scientific Instruments 


\title{
Magnetic measurements on the TCV Tokamak
}

\author{
J.-M. Moret, F. Buhlmann, D. Fasel, \\ F. Hofmann, G. Tonetti \\ Centre de Recherches en Physique des Plasmas \\ Association EURATOM - Confédération Suisse \\ École Polytechnique Fédérale de Lausanne \\ CH-1015, Lausanne, Switzerland
}

The TCV Tokamak was designed to create a large variety of plasma shapes. Such a large flexibility requires high precision magnetic measurements with a good spatial coverage. This paper gives a detailed description of the magnetic sensor geometry, fabrication, calibration, the associated electronics and the diagnostic operation and monitoring. A substantial effort has been made to quantify the precision in the measurements and a novel method has been developed to derive corrections in the sensor position and calibration which optimise the consistency of the entire measurement set. Accuracy of $0.5 \mathrm{mWb}$ in the poloidal flux and $1 \mathrm{mT}$ in the magnetic field with a position error of a few $\mathrm{mm}$ have been achieved. 


\section{Introduction}

TCV (Tokamak à configuration variable) was built to study the influence of the plasma shape on tokamak physics and plasma performance $[1,2]$. Planned shapes include limited and open divertor configurations with an elongation up to 3, with upper or lower single null or double null divertor, or even more complex configurations such as doublets. The machine was designed to keep a flexible control on the exact contour of the plasma column and on the divertor leg geometry in all these configurations.

Such a large flexibility has important implications on the required precision and scope of the magnetic measurement set used for both real time control of the plasma shape and discharge parameters and off-line reconstruction of the magnetic topology. A detailed description of the magnetic measurements available on TCV is given here, covering the sensor geometry and location, their fabrication and calibration, the electronics providing the required signal processing, the operation of the diagnostic and its continuous monitoring which taken together guarantee the quality of the measurements. A substantial section is dedicated to the effort made to quantify the precision of the measurements and to derive the corrections which optimise their consistency. The conclusion is written as an objective evaluation of the system, listing not only the positive aspects but also the difficulties encountered and the various points for which small or significant improvements could or should be brought.

\section{Layout}

\subsection{Ohmic transformer, shaping and toroidal field coils}

The plasma current induction system of TCV is an air core transformer made of two coil sets, coil A and coils B1, B2, C1, C2, D1 and D2 connected in series, shown in figure 1 . Their positions and numbers of turns have been chosen to minimise the magnetic field inside the vacuum vessel. Each set has a separated power supply, labelled $\mathrm{OH} 1$ and $\mathrm{OH} 2$. 


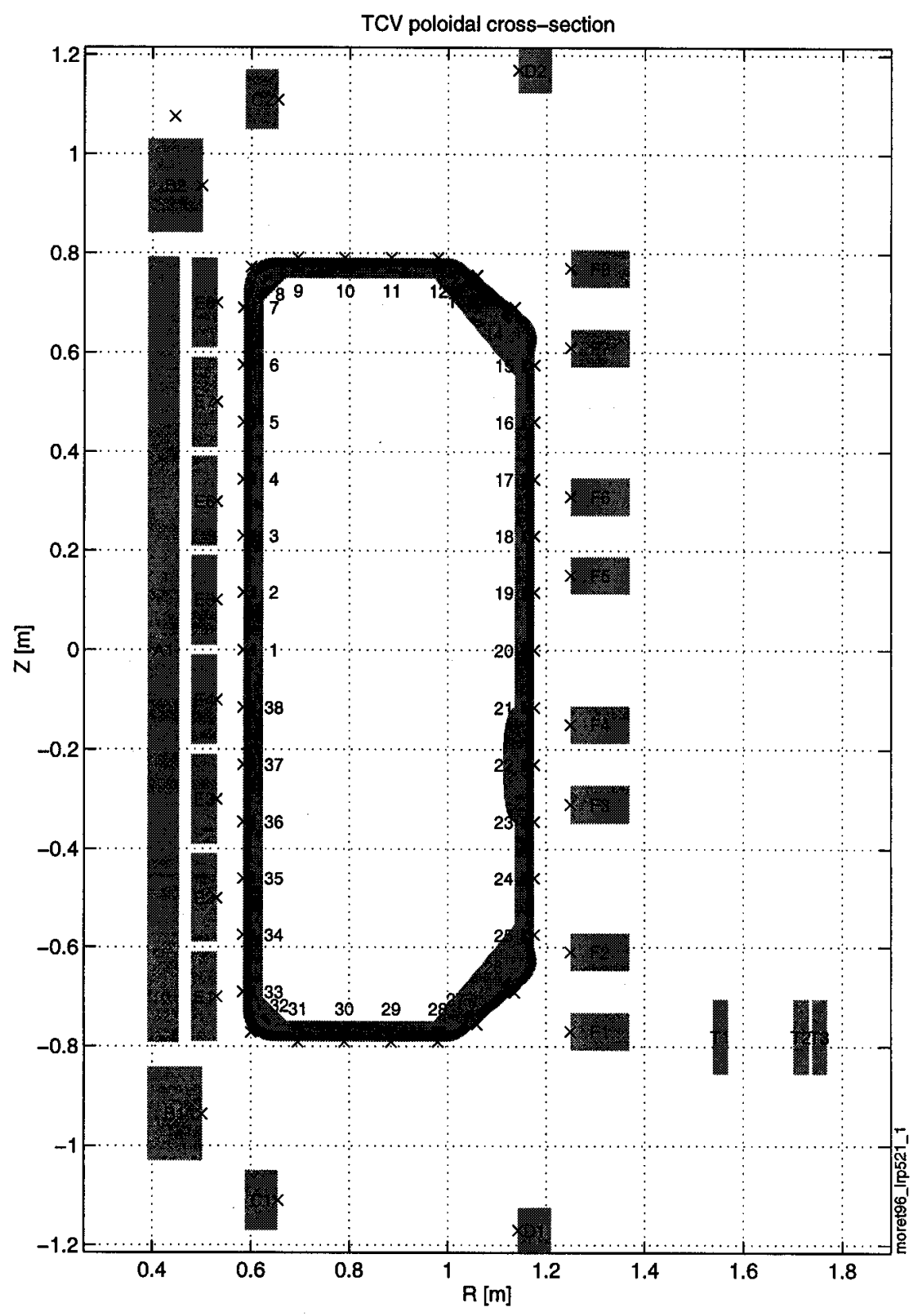

Fig. 1 TCV poloidal cross-section showing the ohmic transformer coils $A, B$, $C$ and $D$, the shaping coils $E$ and $F$, the toroidal field coil connections $T$, the poloidal flux loops $(x)$ and the magnetic field probes plotted as to scale rectangles in the vacuum vessel.

Flexible shape control requires a large number of poloidal coils and is provided on TCV by two stacks of eight coils located on the high and low field side of the vacuum vessel, labelled respectively E1 to E8 and F1 to F8 (figure 1), and connected to 16 independent power supplies. 
The toroidal field is produced by 16 coils surrounding all the ohmic and shaping coils. Of particular interest for the magnetic measurements are the bars between these coils that run toroidally and thus produce a poloidal field. Coils are connected together with $U$ shaped bars to withstand mechanical deformations. The resulting toroidal current can be represented by two coils labelled $\mathrm{T} 1$ and $\mathrm{T} 2$ in figure 1 ; the current follows $26 / 68$ of a whole turn in the first coil and the remaining $42 / 68$ in the second. Return current is carried by the complete turn coil T3.

\subsection{Vacuum vessel}

To accommodate highly elongated plasmas with a large variety of shapes, the vacuum vessel has an almost rectangular cross-section with an aspect ratio of $3: 1$. It is toroidally continuous and is made of stainless steel, $15 \mathrm{~mm}$ thick for the cylindrical parts and $20 \mathrm{~mm}$ thick for the top and bottom parts, giving a toroidal electrical resistance of $45 \mathrm{~m} \Omega$. Such a low resistance helps in passively stabilising highly unstable configurations. The inner wall of the vessel is covered with $24 \mathrm{~mm}$ thick carbon tiles.

\subsection{Magnetic measurement location}

The control and the instrumentation for such a wide range of plasma shapes imply a fairly dense set of sensors. Therefore magnetic probes measuring the component of the poloidal field tangential to the vacuum vessel were mounted inside the vacuum vessel at 38 almost evenly spaced positions. Their exact location and orientation can be consulted in figure 1 and in table I. 4 poloidal cross-sections toroidally separated by $90^{\circ}$ are equipped with such a 38 probe array. The signals from two opposite sections are acquired and used for equilibrium reconstruction. The same signals are averaged to cancel any toroidal asymmetry and fed in real time to the plasma shape and position control system. In addition, two toroidal arrays, located in the equatorial midplane at probe position number 1 and 20 and made respectively of 8 and 16 probes, are installed for MHD mode analysis.

Poloidal flux loops are wound on the outside of the vacuum vessel. Their position with respect to each of the 38 magnetic field probes was chosen to fa- 
cilitate flux extrapolation towards the plasma last closed flux surface by pairing one magnetic probe and flux loop (see figure 1 and table I). In addition all ohmic transformer coils, except the A coil which by construction remains rather inaccessible and all shaping coils were equipped with a poloidal flux loop (figure 1). 


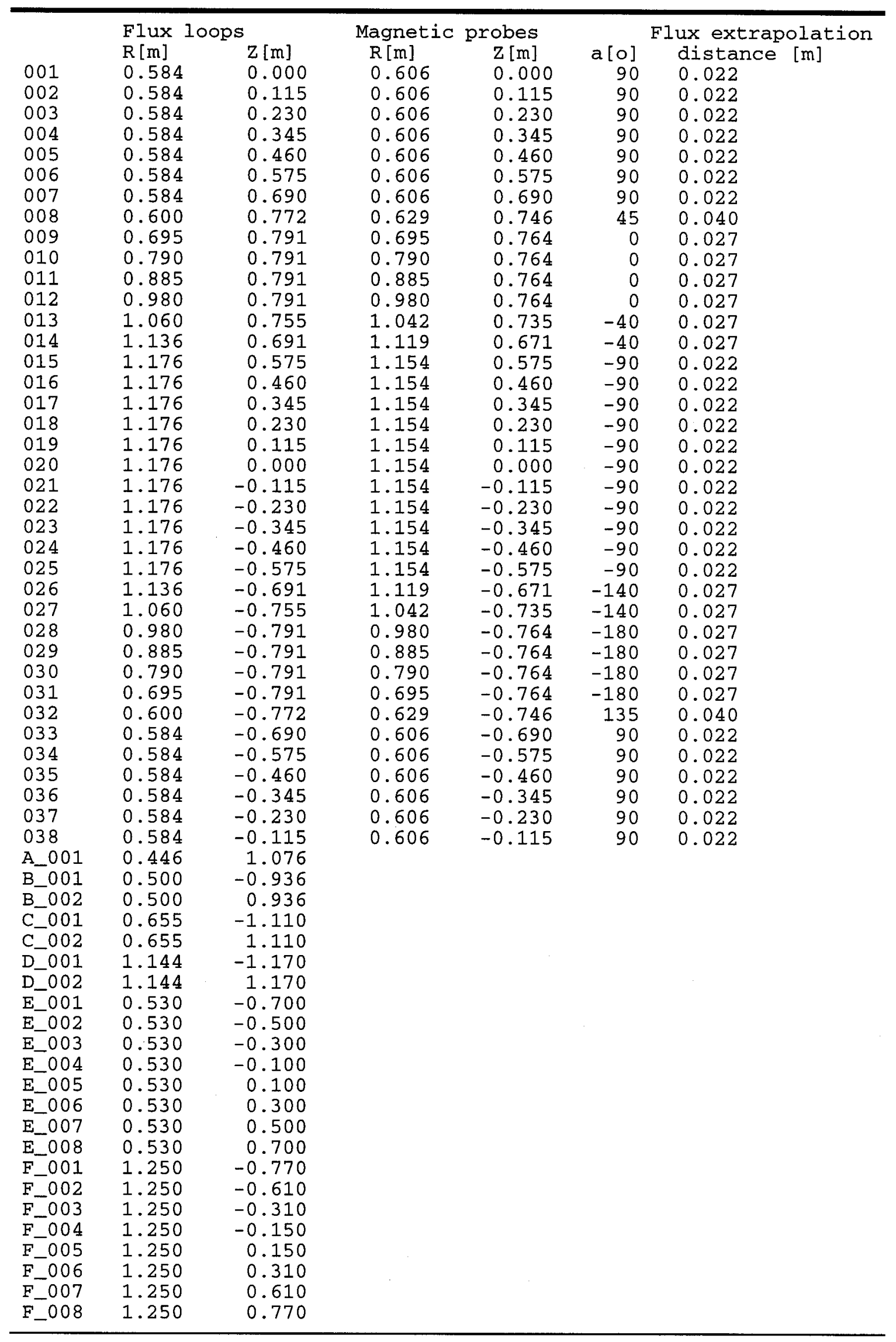

Table I. Flux loops and magnetic probes geometric location. 


\section{Coil current measurement}

Coil current are measured with LEM modules. These are basically iron transformer whose secondary current is adjusted to cancel the magnetic field produced by the bus bar current. The field is measured with hall effect sensor. Modules must be carefully placed away from knees in the bus bars or other bus bars to avoid parasitic field effect. The precision of the measurement quoted by the manufacturer is typically $0.25 \%$ of the nominal current, which corresponds to $100 \mathrm{~A}$ for the $\mathrm{OH}$ currents and $20 \mathrm{~A}$ for the $\mathrm{E}$ and $\mathrm{F}$ currents.

\section{Poloidal flux loops}

\subsection{Construction}

Vessel flux loops are made of one turn of $1 \mathrm{~mm}$ diameter THERMO$\mathrm{COAX}^{\circledR}$, mineral insulated coaxial wire, compatible with the $400^{\circ} \mathrm{C}$ baking capability of the vacuum vessel. Coil flux loops are made of one turn of adhesive copper band applied directly on the coil outer insulation.

Not all of these vessel flux loops can run on a constant radius around the vacuum vessel and some of them must bypass the ports. These deviations are kept as short as possible and when possible distributed equally either side of the ideal path (figure 2). Because of the presence on the top and the bottom of the vessel in sector 11 and 12 of ports with a large poloidal extension, 6 loops, number $10,11,12,28,29$ and 30 , were chosen not to cross these sectors and to return following almost the path of loops $11,12,13,27,28$ and 29 respectively. Thus these partial flux loops do not measure the full poloidal flux but only the flux enlaced by one loop and its neighbour. 

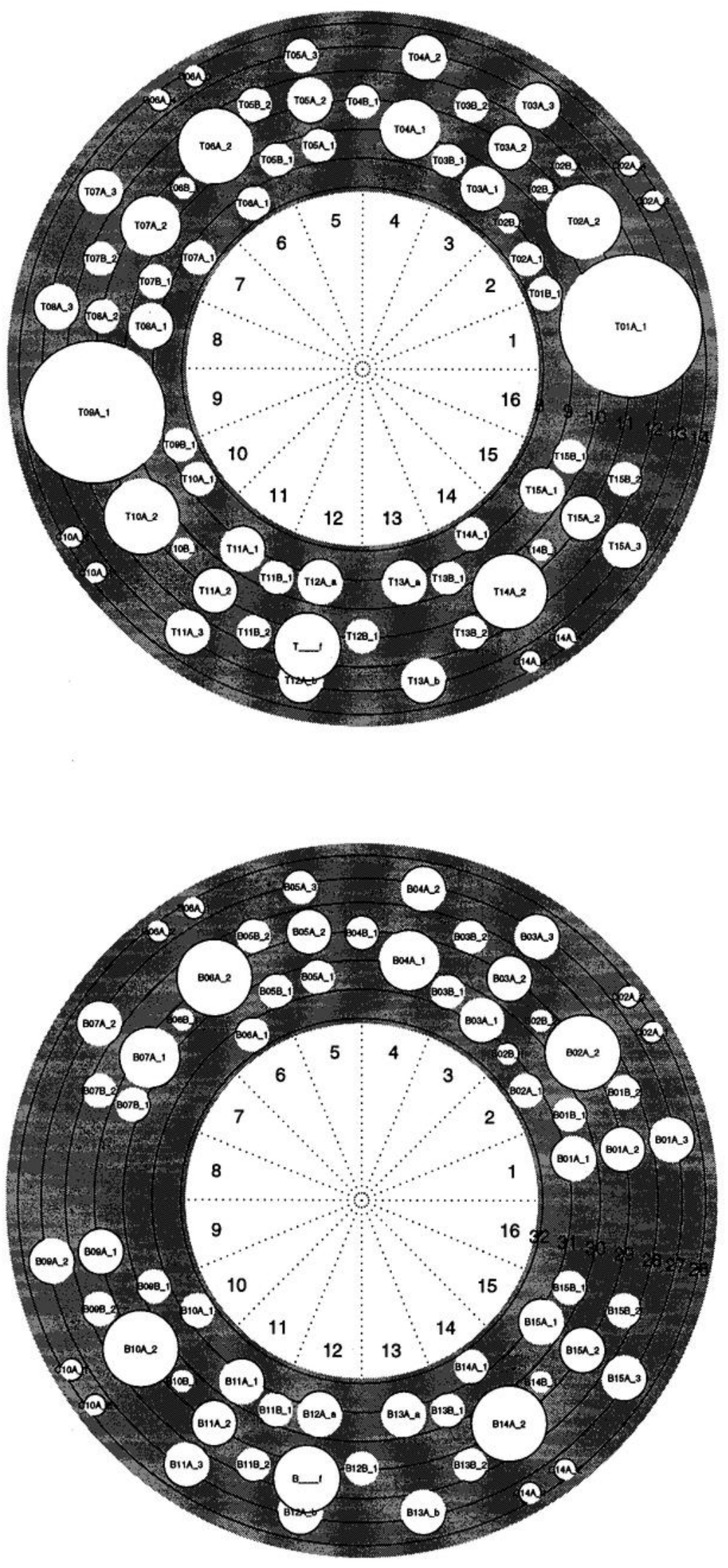


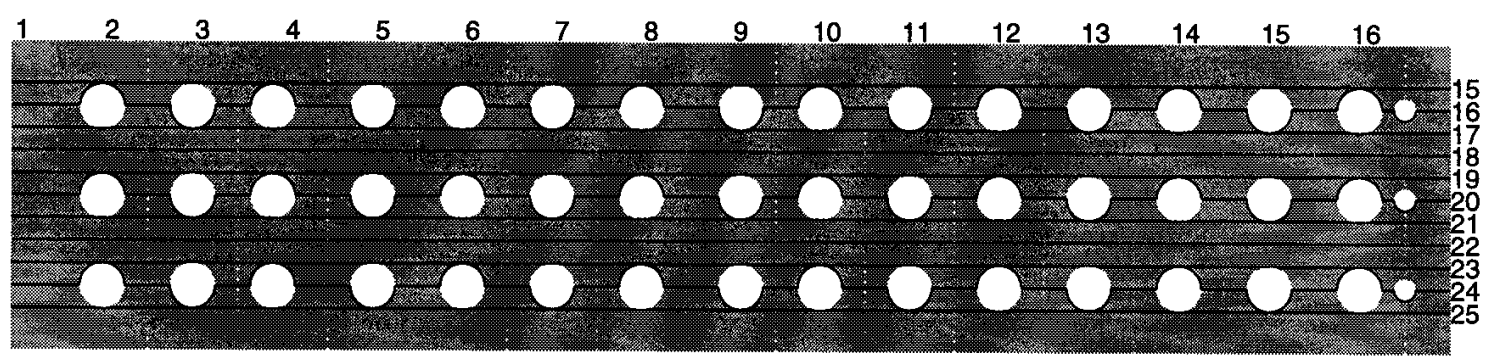

Fig. 2 Arrangement of the flux loops wound on the vacuum vessel and their path around the ports.

\subsection{Signal selection}

Only the coil loops and a selected set of 10 loops almost evenly distributed around the vessel $(1,4,8,13,17,20,23,27,32,36)$ are used to measure the full poloidal flux. These signals are dominated by the ohmic transformer flux and in order to maintain a good dynamic range in the amplifying electronics and data acquisition, the signal from the reference loop number 1 is passively subtracted from the signal of the vessel loops number 2 to 38 , except the 6 partial loops.

The exact area of each loop segment can be evaluated from the constructional dimensions. Under the assumption that the normal magnetic field is constant between two adjacent loops, coefficients can then be derived which allow to compute the flux measured by each loop as a linear combination of ideal fluxes from perfect loops. These coefficient can be packed in a square matrix $T_{r f}$ defined by

$$
\psi_{r}=T_{r f} \psi_{f}
$$

where $\psi_{f}$ is the array of the 38 ideal fluxes and $\psi_{r}$ the array formed by the reference full flux from loop number 1 and the 37 relative fluxes. The inverse transformation matrix is simply defined as $T_{f r}=T_{r f}^{-1}$ and used in converting the measured fluxes in ideal fluxes:

$$
\Psi_{f}=T_{f r} \psi_{r}
$$


The elements of both these matrices are listed in Table II and III. A better estimation of these transformation matrices based on real measurements will be given in section 8.4 .

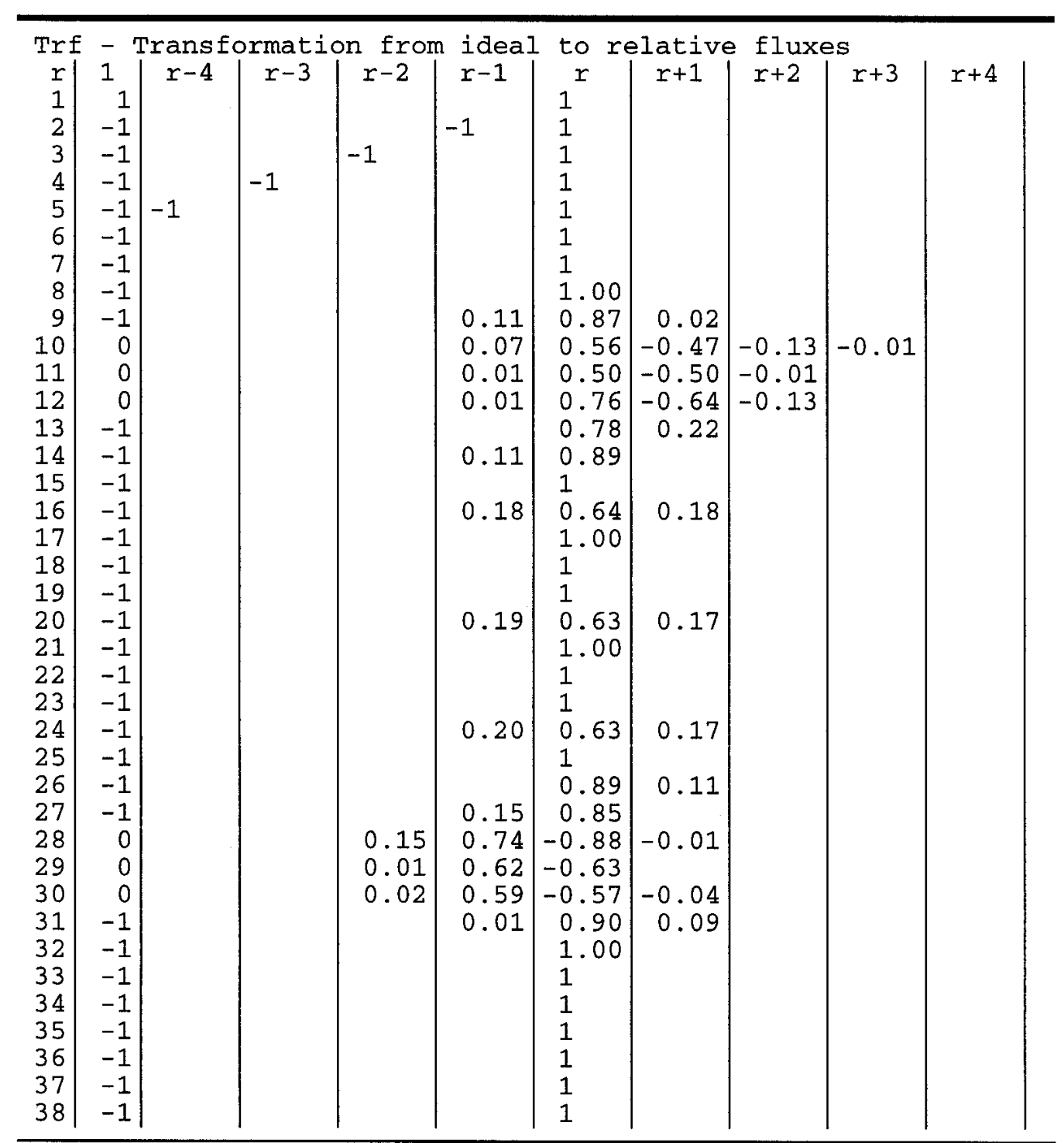

Table II. Matrix coefficients of the transformation from ideal to relative fluxes. 


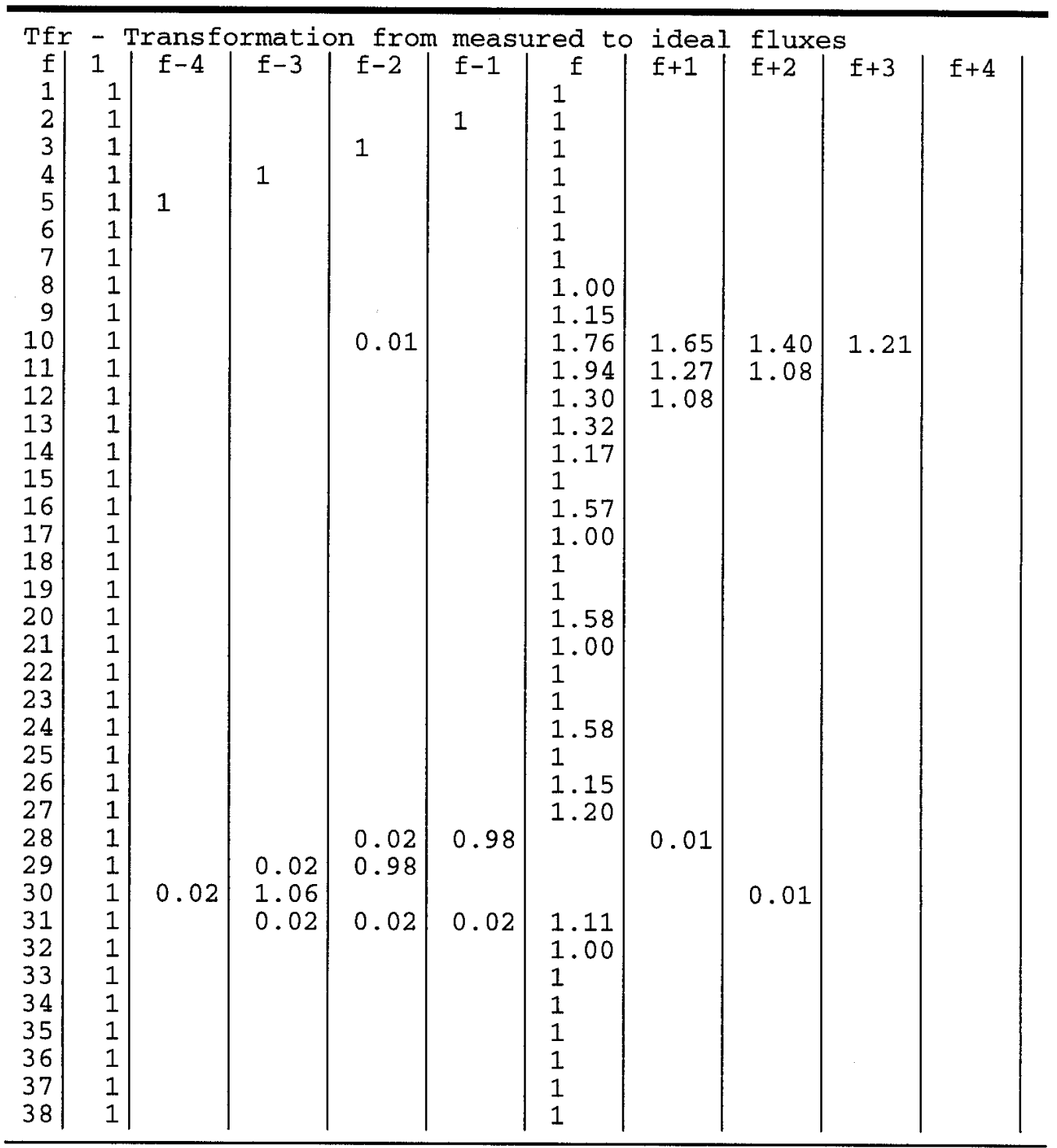

Table III. Matrix coefficients of the transformation from relative to ideal fluxes.

\section{Magnetic probes}

\subsection{Construction}

The design of the magnetic probes was subject to five technical constraints:

1. They should be bakeable to $400 \mathrm{C}$.

2. The maximum thickness of the protection tiles was limited to $24 \mathrm{~mm}$ to allow the plasma to be close to the wall and the passive stabilisation by the vessel eddy currents to be efficient. On the other hand the minimum tile thickness was estimated from thermal load calculation to be $12 \mathrm{~mm}$. 
This leaves $12 \mathrm{~mm}$ in which to house the magnetic probes.

3. The minimum required signal level implies an effective probe area of a few hundred $\mathrm{cm}^{2}$. Details on the expected signal level can be found in section 6.4 .

4. The signal cables of a 38 probe array has to fit in 4 signal feed-through. Thus the corresponding 20 cables torch had to be small enough to run behind the tiles.

5. The frequency response of the probe should be adequate to allow the same probe to be used for MHD activity studies, that is a few $10 \mathrm{kHz}$. More on the probe frequency characteristics also to be found in section 5.3.

The selected solution is a moulded ceramic core (figure 3 ) on which two layers of 20 turns each are wound. The wire is made of $0.5 \mathrm{~mm}$ diameter THERMOCOAX ${ }^{\circledR}$ with a copper central conductor and an Inconel shield. The wire is continuous from the probe to outside the vessel so that no connection is required inside the vessel and the vacuum-tight shield does not need to be broken. Each probe is spring loaded against special fixation rails welded onto the vessel walls and protected by a graphite tile (figure 3 ).

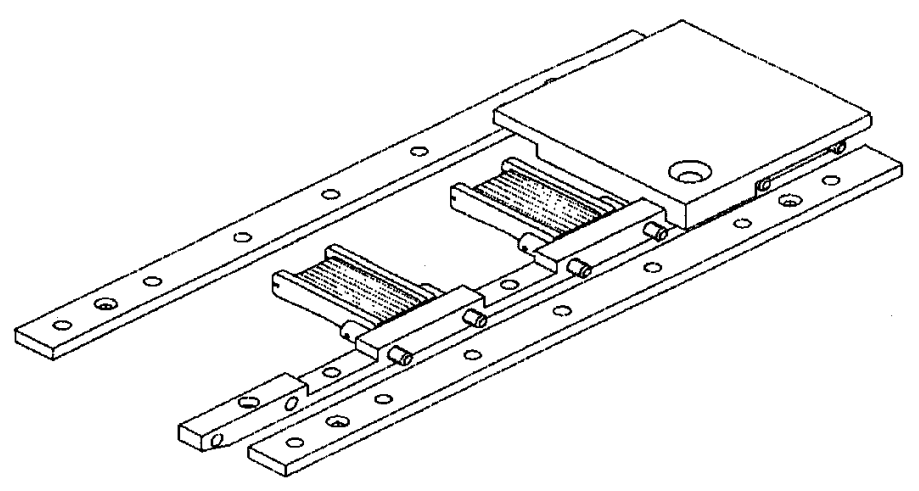

Fig. 3 Drawing of the moulded ceramic core and the magnetic probe assembly on the vacuum vessel.

\subsection{Calibration}

The aimed precision of the measurement from the magnetic field probes is a fraction of $1 \%$. To obtain this, each element must be calibrated with a pre- 
cision of the order of $0.1 \%$. The probe effective area can not be known within these limits, mainly because the wire has a very small curvature and can not be tighly wound. Thus the area of each probe has to be calibrated separately.

This was done using a pair of Helmoltz coils $0.3 \mathrm{~m}$ in diameter separated by $0.15 \mathrm{~m}$. In the centre of this coil, the axial magnetic field varies by less than $0.1 \%$ over the volume occupied by the probe and this variation has been accounted for in the calibration process. Position errors and misalignment with respect to the Helmoltz coils produce an error of $0.1 \%$. The transfer function between the current in the Helmoltz coil and the voltage on the probe is measured between $125 \mathrm{~Hz}$ and $16 \mathrm{kHz}$. This function is then extrapolated to $0 \mathrm{~Hz}$ (see section 5.3 for details) and the probe area derived. Figure 4 shows the distribution histogram of the area, which ranges from $346 \mathrm{~cm}^{2}$ to $366 \mathrm{~cm}^{2}$.

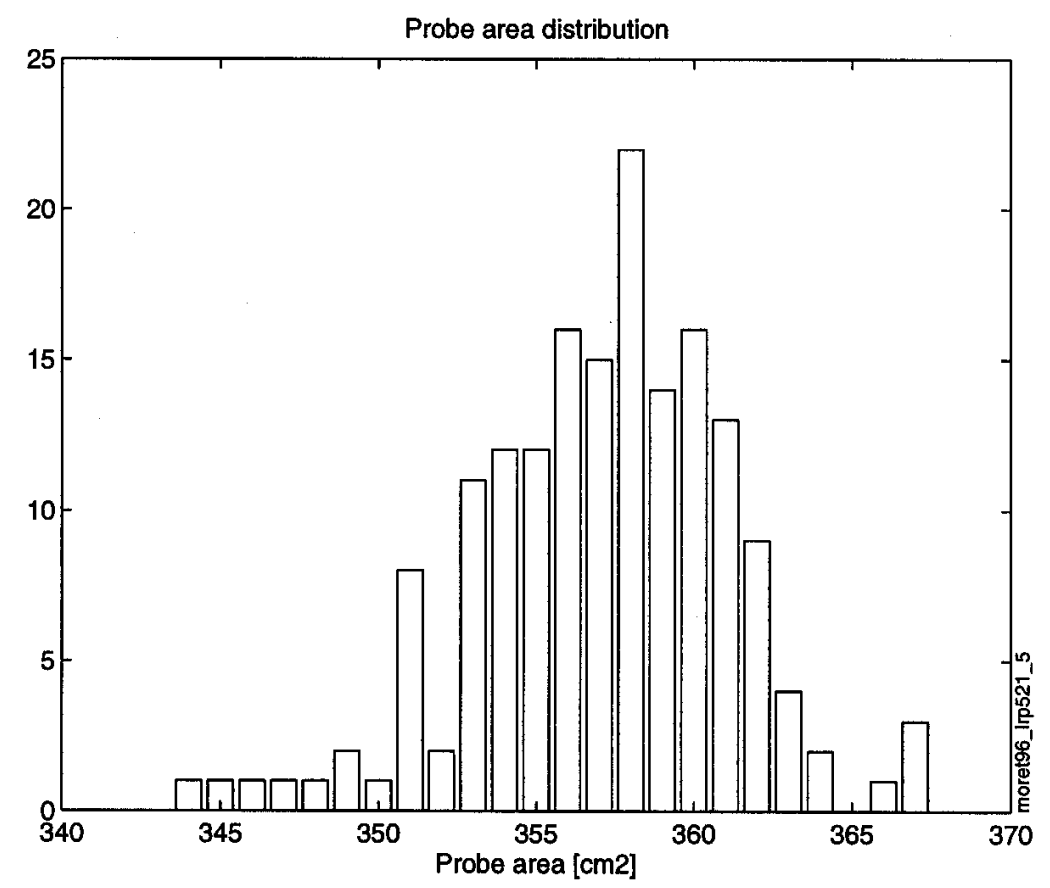

Fig. 4 Histogram of the calibrated magnetic probe area for the 168 probes.

\subsection{Frequency response}

The measured frequency response up to $16 \mathrm{kHz}$ plotted in figure 5 shows that already in this frequency range it can not be reduced to a simple first order transfer function. Therefore the equivalent probe circuit was chosen to include also the capacitance of the cable and shield circuit, as sketched in figure 
6. The transfer function between the magnetic field and the probe voltage is then given by

$$
H_{p}=-\frac{U_{p}}{s B}=\frac{A_{p}}{1+s\left(\frac{L_{s}}{R_{s}}+R_{p} C_{p}\right)+s^{2} R_{p} C_{p}\left(\frac{L_{p}}{R_{p}}+\frac{L_{s}}{R_{s}}\right)}
$$

where the symbols correspond to those in the figure. The measured data points are consequently fitted to a transfer function of the form

$$
H_{p}=\frac{A_{p}}{\left(1+s \tau_{1}\right)\left(1+s \tau_{2}\right)}
$$

using an algorithm described in [3] to extract the probe area $A_{p}$, the probe cutoff frequencies $1 /\left(2 \pi \tau_{1}\right)$ and $1 /\left(2 \pi \tau_{2}\right)$. Typical values for these frequencies are $134 \mathrm{kHz}$ and $390 \mathrm{kHz}$. The statistical error on the estimation of the probe area by this fitting is typically $0.05 \%$.

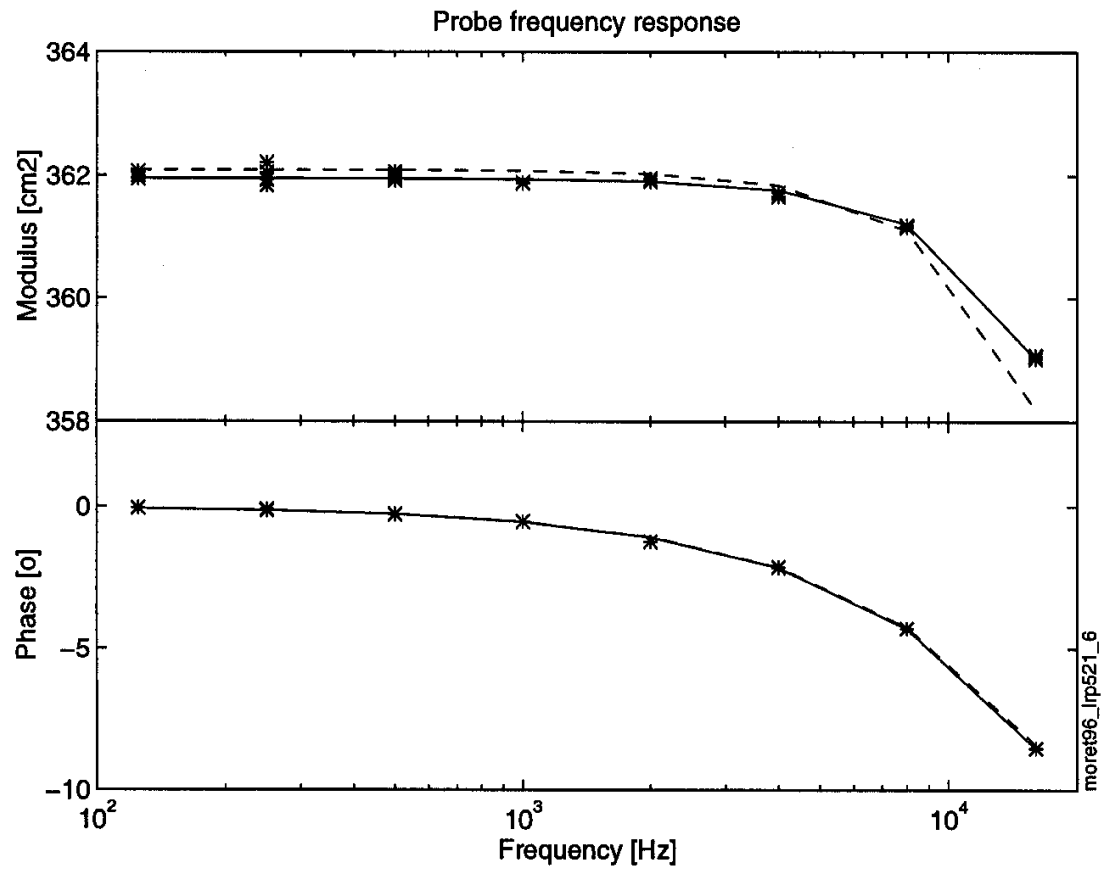

Fig. 5 Magnetic probe frequency response. (stars: calibration measurements; dotted line: fitted first-order transfer function; solid line: fitted second-order transfer function. 


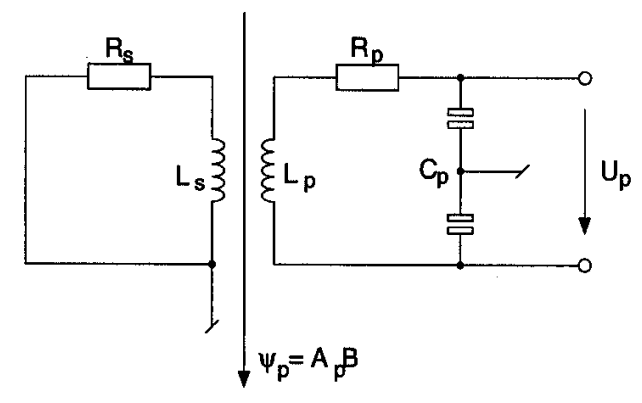

Fig. 6 Equivalent circuit of the magnetic probes.

\section{Signal processing}

\subsection{General block diagram}

Figure 7 shows the block diagram for the signal processing of each type of signal. Signals from the LEM modules measuring the coil currents are simply amplified. The flux loop signals are either amplified to be used as loop voltages or integrated to obtain the poloidal flux. Magnetic probe signals need first to be preamplified near the machine before the long transmission line. They are also either amplified to be used as the time derivative of the field or integrated to obtain the field itself. Some magnetic probe signals are also attenuated to study fast events such as disruptions. Each signal is low pass filtered before being acquired or fed to the shape and position control system. Each of these elements will now be described. 


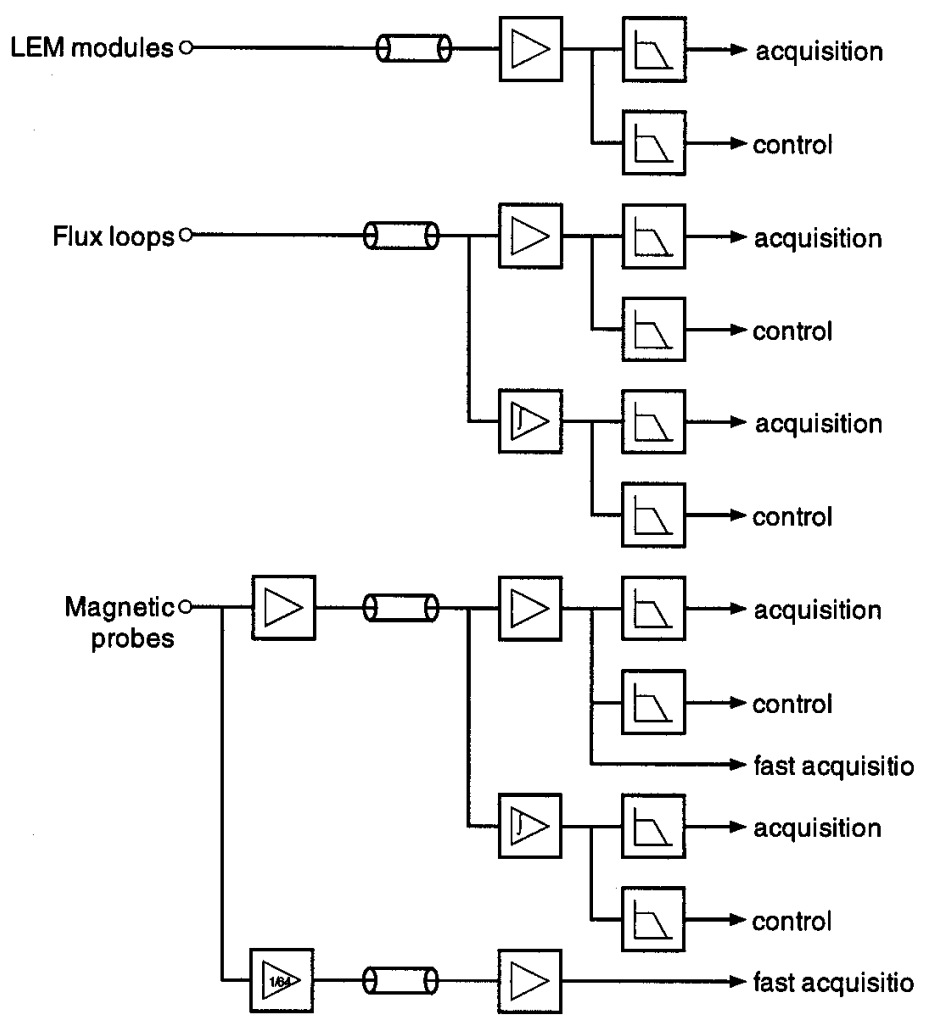

Fig. 7 Block diagram of the amplifying electronics for all magnetic signals.

\subsection{Amplifiers}

The signal fed in an amplifier first passes through a first-order low-pass filter at $80 \mathrm{kHz}$ which is latter compensated in the second amplifier stage. It is then amplified by a programmable amplifier with gains of $1 / 4,1 / 2,1$ or 2 , and then by a second stage with a gain of 1 or 16, giving an overall gain range of $1 / 4$ to 32 . The amplifiers have a common mode rejection of $40 \mathrm{~V}$, an automatic compensation of the global offset and a saturation detection that can be read after each tokamak discharge.

\subsection{Integrators}

The signal fed into an integrator first passes through a first-order lowpass filter at $3.5 \mathrm{kHz}$ in order to remove spikes in the signal that would saturate the input stage. It is then amplified with gains of $1 / 4,1 / 2,1$ or 2 , and integrated with a time constant of $12.5 \mathrm{~ms}$ or $200 \mathrm{~ms}$, giving an overall integration time constant from $6.25 \mathrm{~ms}$ to $800 \mathrm{~ms}$. The feedback circuit of the integrating stage compensates the low-pass input filter so that the overall 
transfer function is that of a perfect integrator. The integrators have the same common mode rejection, offset compensation and saturation detection as the amplifiers.

\subsection{Magnetic probe amplifying chain}

The magnetic probe signal amplification needs particular care because of the large frequency spectrum and the wide variety of signals that they detect. The low frequency signal components that come from the coil or the plasma current are of the order of $10 \mathrm{mV}$. Mirnov oscillations with a typical amplitude of a few percent of the poloidal field at $10 \mathrm{kHz}$ give a $10 \mathrm{~V}$ signal. In addition, fast MHD events have been observed with spikes up to $300 \mathrm{~V}$. Therefore, in order to prevent saturation of the preamplifier, but maintain a reasonable signal level, it was necessary to precede the preamplifier by a first-order passive filter with a cut-off pole at $100 \mathrm{~Hz}$ and a zero at $3.5 \mathrm{kHz}$, giving an attenuation factor of $\mathbf{3 5}$ for the high frequency signals. The transfer function of this filter is combined with the input stage filter of the integrator and the integrating stage itself to give a global transfer function of a perfect integrator, as seen on figure 8. Differences in zero-pole cancellation produce no error in steady state conditions and a dynamic relative error at most equal to the relative error in the zero-pole pair divided by the ratio between the integration time constant and that of the implied pair. This ratio is typically 20. 


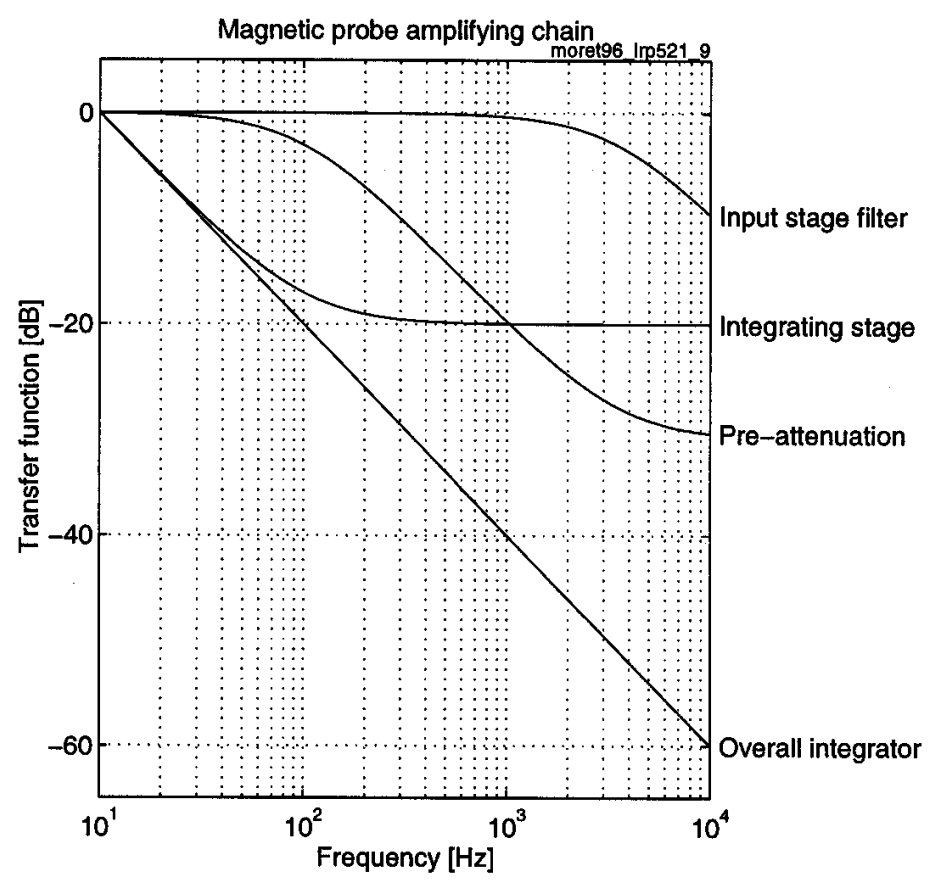

Fig. 8 Amplitude of the transfer function of the successive stages of the magnetic probe amplifying chain.

\subsection{Filters}

Filters are made of second-order Chebychev, $0.1 \mathrm{~dB}$ ripple, low-pass cells with a programmable cut-off frequency from $1 \mathrm{kHz}$ to $25 \mathrm{kHz}$. Anti-aliasing filters consist of two cells usually set to half the sampling frequency. This yields a group delay close to 0.7 sample at low frequency, increasing to almost 2 samples at half the sampling frequency (figure 9). The signals used for real time shape and position control are processed by one cell filter to avoid a too large phase lag that would impair fast instability feed-back. 


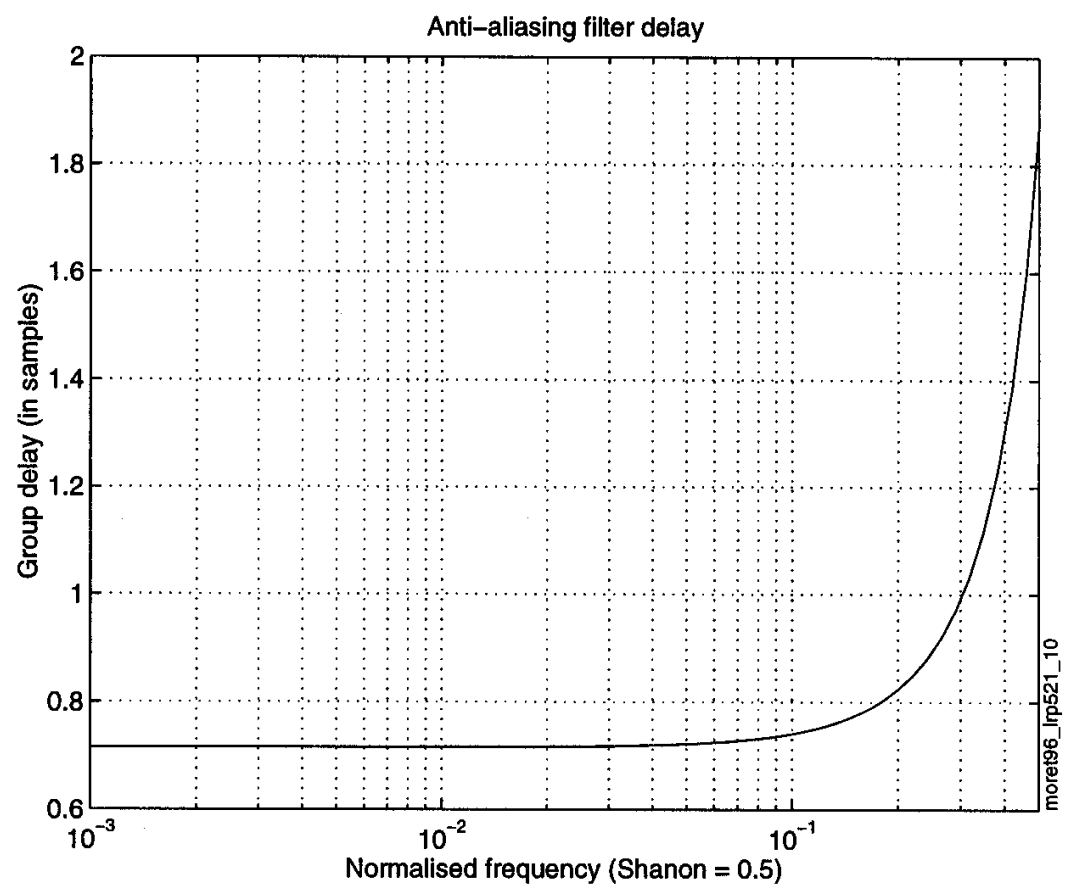

Fig. 9 Group delay of the anti-aliasing filter.

\subsection{Calibration}

Each element of the amplifying chain has been calibrated separately to fulfil the following two goals: get a very precise value of the DC gain and measure the transfer function in the relevant frequency range. To do this an appropriate test signal has been programmed in a wave generator and fed into the element to be tested. Then both input and output are recorded in an ADC. This test signal is a superposition of sine waves:

$$
\sum_{n=1}^{N}(-1)^{n} \sin \left(w_{n} t\right)
$$

The $w_{n}$ are chose between $5 \mathrm{~Hz}$ and $20 \mathrm{kHz}$ with a separation of approximately one third of an octave. The exact frequency value however is selected so that the test signal has a periodicity which is an integer submultiple of the acquisition duration, which means that it fulfils the relationship:

$$
\frac{2 \pi}{w_{n}}=\frac{K T}{k}
$$


where $K$ is the total number of samples, $T$ the sampling period and $k$ an integer. The sign of each sine component is alternate to avoid signal pile up at time $t=0$. Typical signal length used is $128 \times 1024$ samples. The transfer function is then given by the ratio of the fast Fourier transform of the output to that of the input, where only the peaks of the spectrum have been retained (figure 10). The wave generator and the acquisition are synchronised to avoid peak broadening. No window is required for the Fourier transform since the test signal was selected with the appropriate periodicity.

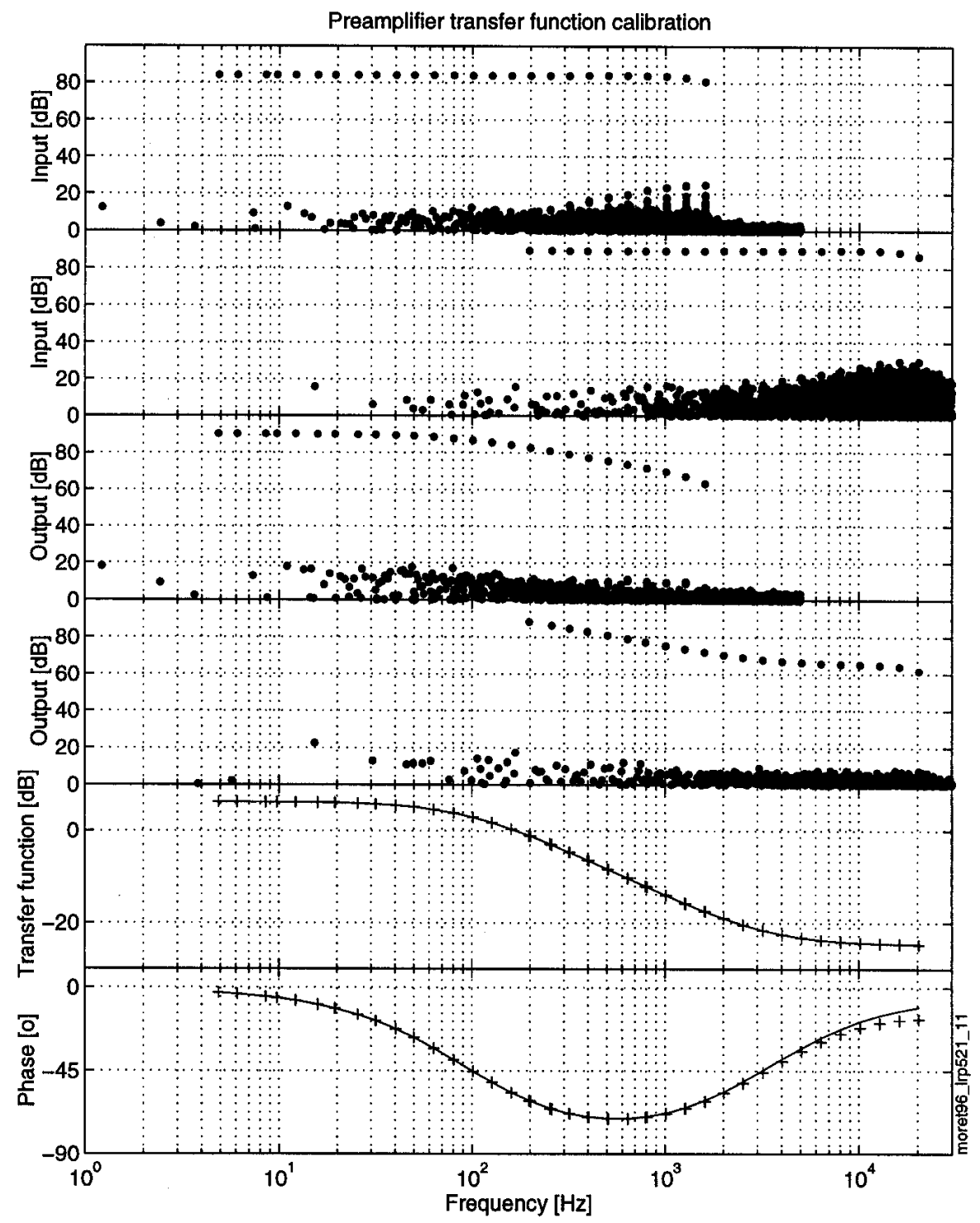

Fig. 10 Amplifying electronic calibration. From top to bottom: input signal spectrogram; output signal spectrogram; ratio of the output to the input peaks together with the low order fitted transfer function. 
A reduced order transfer function is then fitted to the data points, firstly to extrapolate it to zero frequency to get the DC gain, and secondly to express it in terms of zero and poles, a form that can be used for further compensation of the signal (figure 10).

\subsection{Cabling}

Signal transmission from the machine area to the electronic bays uses 12 twisted differential pair cables with individual pair shielding. Cable length varies from 30 to $50 \mathrm{~m}$. The sensors and the preamplifiers located near the machine are connected to the vessel earth. Earth separation is placed on the entry of the electronic bays together with a $50 \mathrm{~V}$ protection spark gaps.

\section{Diagnostic operation}

\subsection{Control and acquisition}

All preamplifier, amplifier and integrator gains, as well as the filter cutoff frequencies are remotely controlled via the TCV control system. The same system monitors and archives possible saturations in the amplifying chain so that machine operators are warned of inadequate gain setting and invalid acquired data is flagged. This represents an overall set of about 2000 variables to be controlled and monitored.

Signal acquisition is performed by 12 bit ADC's usually at a sampling frequency of $2 \mathrm{kHz}$ during the 1 to $2 \mathrm{~s}$ pulse duration. These represents of the order of 1 million samples which are archived in a compressed format by the MDS+ data acquisition system [4] in a 0.5 Mbyte file.

\subsection{Signal verification}

The whole detection chain of each signal, from the probe, cabling, electronics to the acquisition and the real time control system, is checked every morning with a specially designed test. This is done by consecutively firing each ohmic transformer, shaping and toroidal field power supplies. The recorded signals are shown in figure 11 . The expected poloidal flux and magnetic field produced by these poloidal currents are computed for each loop and 
probe and compared to the real signal to detect any fault in the diagnostic. The current time evolutions are slow enough and vessel eddy currents can be neglected.
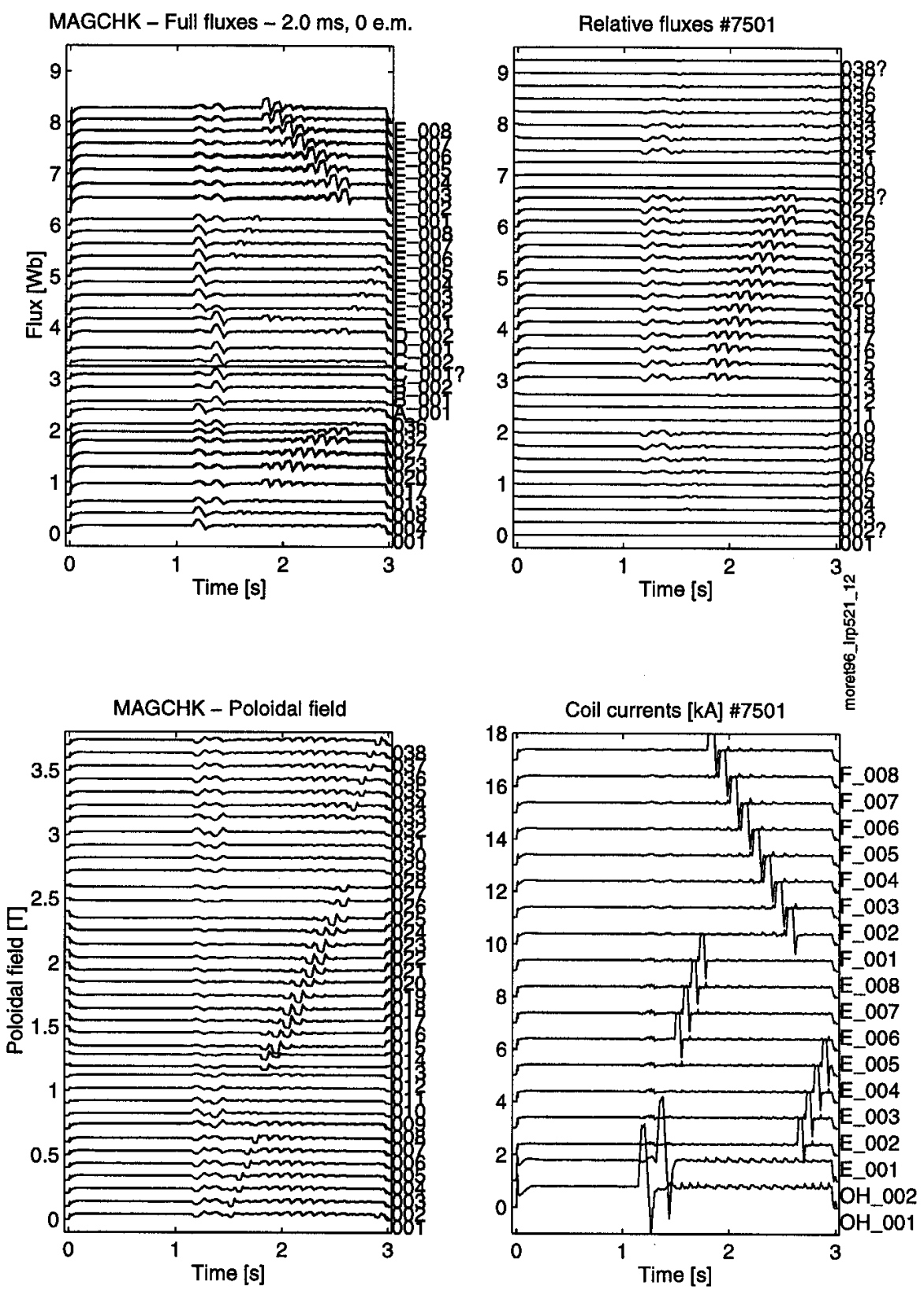

Fig. 11 Test signals used for daily check of the entire diagnostic.

\section{Consistency and precision}

\subsection{Expected, required and measured precision}

The entire diagnostic was built to guarantee a precision better than $1 \%$. Coil current measurements have an error much better than $1 \%$ of the nominal 
current, leading to a tolerable error for the used current range. Flux measurements do not require any detector calibration and suffer only from electronic errors; they should therefore be the most precise. The magnetic field measurements are the most complex and therefore the least precise. As described before, the error in the probe area calibration may reach $0.5 \%$. The amplifying chain is also more complex since it is done in two stages. Nevertheless an overall precision better than $1 \%$ looks still feasible. These precision ranges are thought to be largely sufficient for real time control of the plasma as well as for equilibrium reconstruction, especially because of the large number of measurements available.

A more detailed study of the precision of the magnetic measurements would however help in using the data safely and optimaly. To this purpose the following experiments are carried out: each ohmic transformer coil set, shaping coil and toroidal field coil system are separately powered by a given current. This yields for each magnetic signal a set of 19 independent measurements that should provide basis to infer the level of correctness of the diagnostic. Compared to the daily test designed to detect only the rough faults in the system, these experiments have been given special care to ensure low and adequate experimental errors:

1. The only current or in other words the only flux and magnetic field source must be the powered coil. In particular the experiment must be long and stationary enough to make sure that the eddy currents in the vessel or any other massive conductive structure of the machine has vanished. To obtain flux and magnetic field measurements with a precision of $0.1 \mathrm{mWb}$ and $0.1 \mathrm{mT}$ respectively, the current in the coil should not derive for more than $100 \mathrm{~A}$ in 1 second. In practice the current in the coils has almost a 1 second flat top during which the current is controlled within 10A, as shown by the example of figure 12. The same figure shows that the drift is also negligible compared to the sought precision for the flux loops and the magnetic probes.

2. The gains in the amplifying chain must be chosen to use the full available dynamic range because the required precision corresponds to $10 \mathrm{mV}$ for a $10 \mathrm{~V}$ signal or one quantification level in the digitised data. 


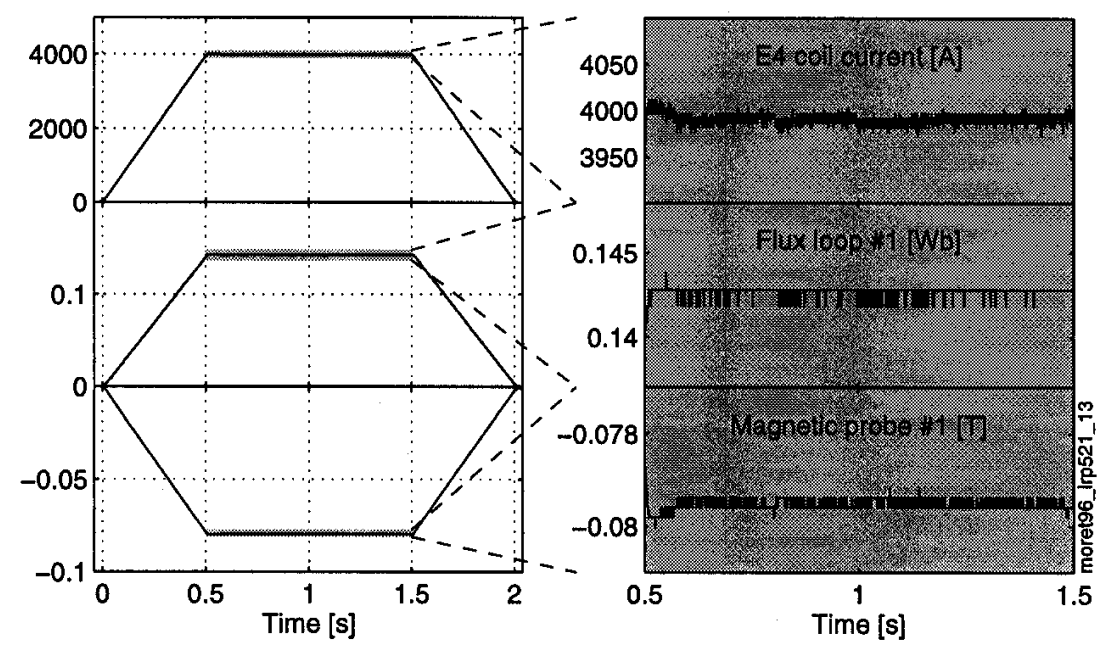

Fig. 12 Experiments used to check the consistency of the magnetic data set. The figure shows the current wave form, one typical flux signal and one typical magnetic field signal.

In addition the signals are time averaged to reduce noise and statistical error. The experimental values of the fluxes $\psi_{f}$ and the magnetic fields $B_{m}$ are then compared to the expected values obtained from the geometrical dimensions and position of the coils and the sensors:

$$
\begin{aligned}
& \psi_{f}=M_{f a} I_{a}, \\
& B_{m}=B_{m a} I_{a} .
\end{aligned}
$$

Here $M_{f a}$ is the mutual inductance between the coil and the flux loop, $B_{m a}$ the tangential magnetic field at the magnetic probe location produced by a unit current in the coil and $I_{a}$ the coil current. In the computation of these quantities, each coil is divided in its individual turns whose size is typically 1 to $3 \mathrm{~cm}$. The spatial extension of the magnetic field probes along their measuring axis is also taken into account since this modifies the value by almost $1 \%$ for the probes located close to the considered coil. Figures 13 and 14 present this comparison: the expected values $M_{f a}$ and $B_{m a}$ and the differences derived from the measurements:

$$
\Delta M_{f a}=\psi_{f} / I_{a}-M_{f a},
$$




$$
\Delta B_{m a}=B_{m} / I_{a}-B_{m a} .
$$

The conclusion is clear: the discrepancy in the measurements reaches $2 \%$ for the flux and 5\% for the magnetic field, both exceeding the expected and tolerable error. Only these loops with no detours around the ports are included in this exercice, the other loops will be treated separately in section 8.4.

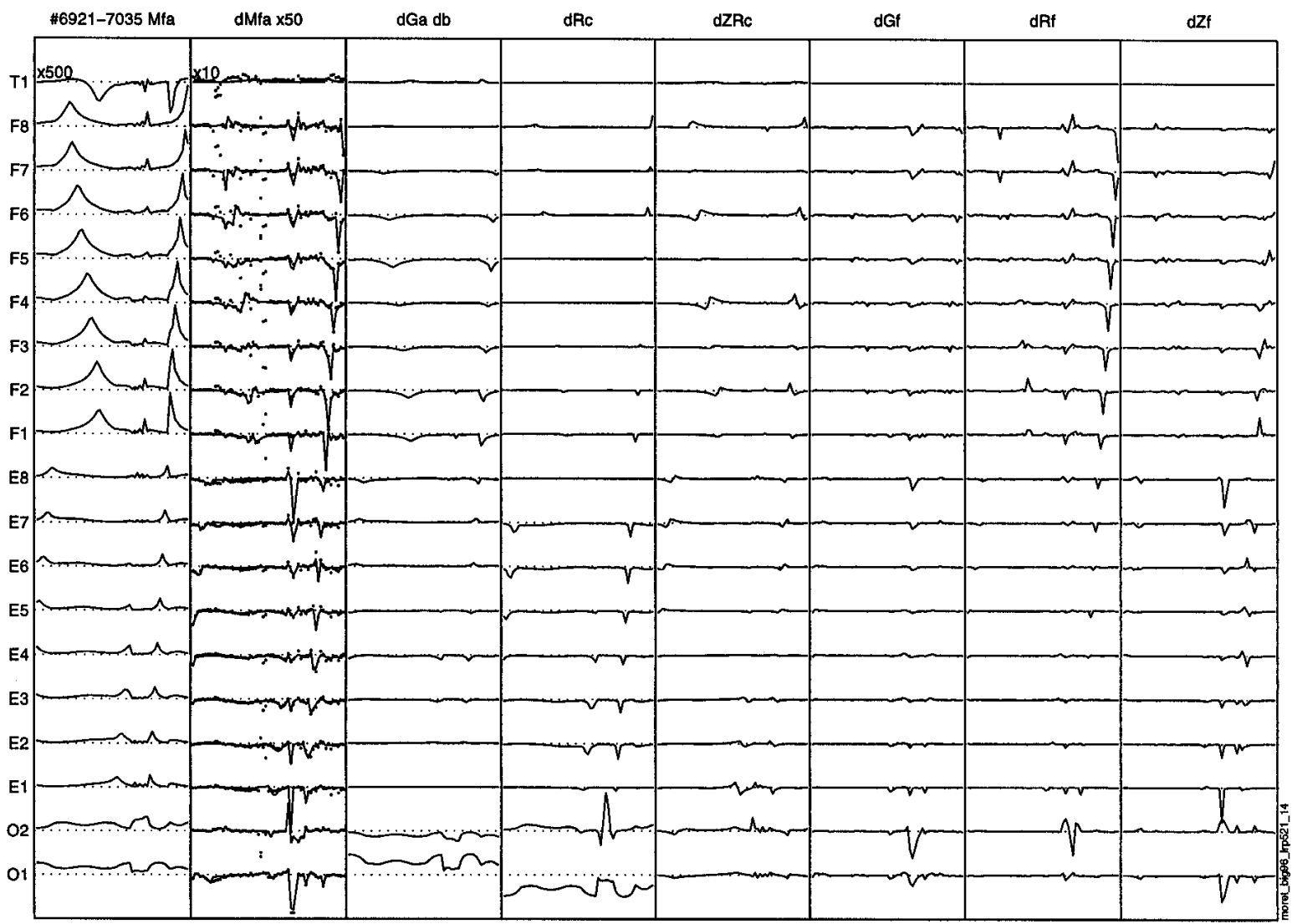

Fig. 13 The expected flux values and their difference with the measurements. Each curve consists of the values as a function of the loop number for each 19 coils or coil sets. $M_{f a}$ are the expected values, multiplied by 500 for the toroidal connections. Dots show the differences with the measurements on an expanded scale $(50 \times)$. The solid line superposed to the dots are the differences explained by the derived corrections on calibration and location of the coils and loops. Follows the individual contribution of each implied correction plotted separately. 


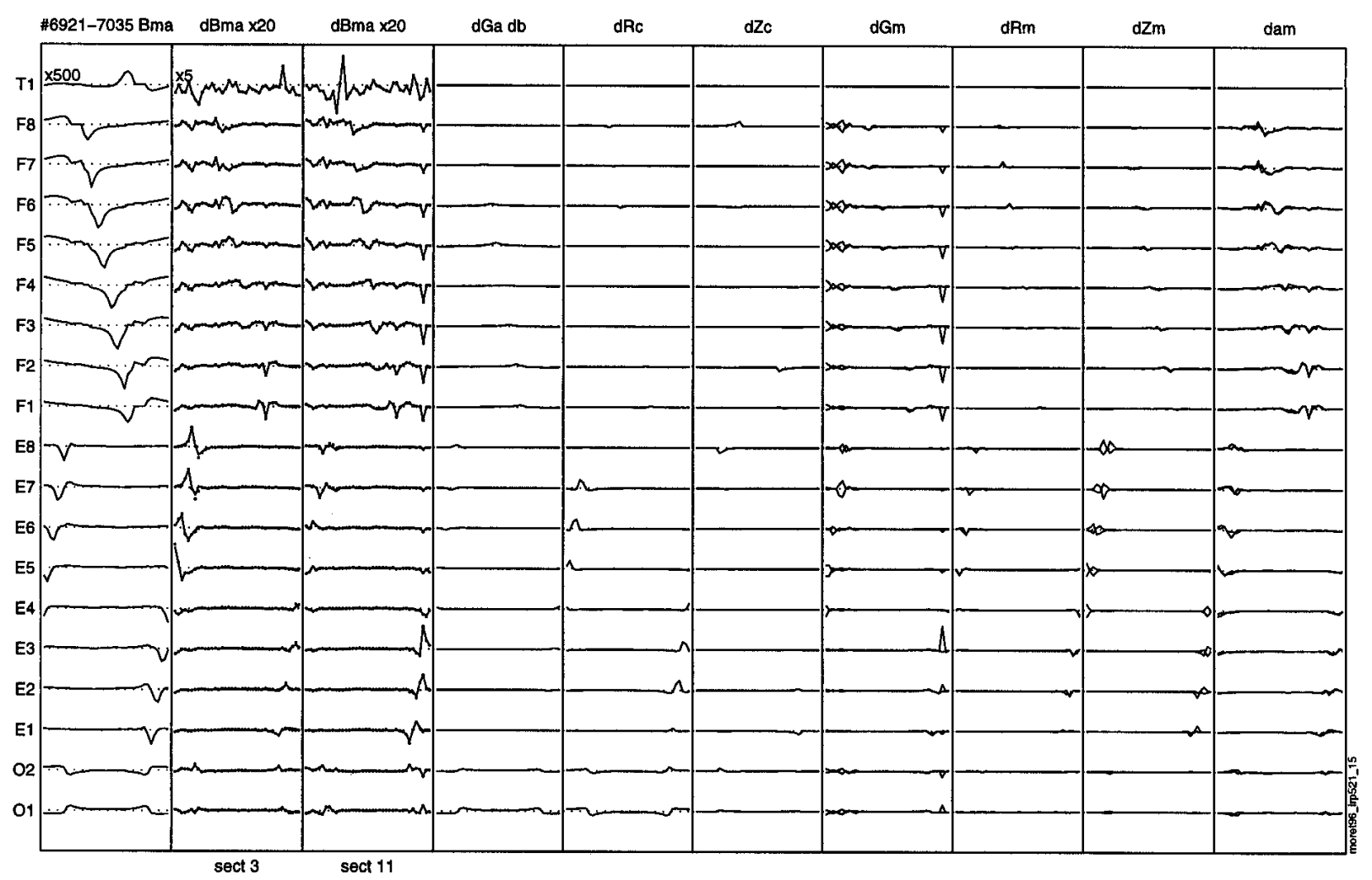

Fig. 14 The expected field values and their difference with the measurements. Each curve consists of the values as a function of the probe number for each 19 coils or coil sets. Bma are the expected values, multiplied by 500 for the toroidal connections. Dots show the differences with the measurements on an expanded scale $(20 \times)$. The solid line superposed to the dots are the differences explained by the derived corrections on calibration and location of the coils and probes. Follows the individual contribution of each implied correction plotted separately.

\subsection{Sources of error}

The explanation for these differences is to be found mainly in small calibration and geometrical errors: the actual location of the coils, flux loops or magnetic probes may not be exactly the design location. Cumulated difference due to small errors may explain the observed discrepancy. Possible sources of error are listed below together with their effect on the flux and magnetic field: Error in the calibration of the coil current measurements. This was specified to be less than $1 \%$. It gives rise to a flux difference of 


$$
M_{f a}\left(\Delta G_{a}\right) I_{a}
$$

where $\Delta G_{a}$ is a relative calibration error. The corresponding magnetic field difference is

$$
B_{m a}\left(\Delta G_{a}\right) I_{a}
$$

Misplacement of the coils. Given the overall size of the machine, this may be of the order of a few $\mathrm{mm}$. A radial and vertical displacement of a coil of respectively $\Delta R_{c}$ and $\Delta Z_{c}$ produces a difference in the flux and in the magnetic field of respectively

$$
\frac{\partial M_{f c}}{\partial R_{c}}\left(\Delta R_{c}\right) I_{c}+\frac{\partial M_{f c}}{\partial Z_{c}}\left(\Delta Z_{c}\right) I_{c}
$$

and

$$
\frac{\partial B_{m c}}{\partial R_{c}}\left(\Delta R_{c}\right) I_{c}+\frac{\partial B_{m c}}{\partial Z_{c}}\left(\Delta Z_{c}\right) I_{c}
$$

Indexing of the coil current has been changed from $a$ to $c$ to treat separately coils which are connected in series to a single power supply. $I_{c}$ and $I_{a}$ are linked by the connection matrix $T_{c a}$.

\section{Error in the calibration of the flux and magnetic field measurements.}

As mentioned before, this error is thought to be much less than $1 \%$ for the fluxes and should never exceed $1 \%$ for the magnetic fields. This yields a difference in the flux and magnetic field of respectively

$$
\left(\Delta G_{f}\right) M_{f a} I_{a}
$$

and

$$
\left(\Delta G_{m}\right) B_{m a} I_{a}
$$

where $\Delta G_{f}$ and $\Delta G_{m}$ are relative calibration errors. 
Misplacement of the flux loops. Given that the TCV vacuum vessel is made of a single machined piece, this error should not exceed some mm. Note however that the thermal expansion of the vessel at $400^{\circ} \mathrm{C}$ is $5 \mathrm{~mm}$ and must be taken into consideration. A flux difference of

$$
\frac{\partial M_{f a}}{\partial R_{f}}\left(\Delta R_{f}\right) I_{a}+\frac{\partial M_{f a}}{\partial Z_{f}}\left(\Delta Z_{f}\right) I_{a}
$$

would be produced by a radial and vertical displacement of the loop of $\Delta R_{f}$ and $\Delta Z_{f}$. Any angular misalignment of the vertical axis of the loop with respect to that of the coils would lead to second order corrections.

Misplacement of the magnetic probes. The same argument on the vessel construction limits this displacement to some mm. An error of $\Delta R_{m}$ and $\Delta Z_{m}$ in the radial and vertical position produces a difference in the magnetic field of

$$
\frac{\partial B_{m a}}{\partial R_{m}}\left(\Delta R_{m}\right) I_{a}+\frac{\partial B_{m a}}{\partial Z_{m}}\left(\Delta Z_{m}\right) I_{a}
$$

Any misalignment of the measuring probe axis in the poloidal plane should also be taken into consideration. A difference in the orientation of $\Delta \alpha_{m}$ would produce a field error of

$$
\frac{\partial B_{m a}}{\partial \alpha_{m}}\left(\Delta \alpha_{m}\right) I_{a}
$$

Since the measured field is given by the combination

$$
B_{r m a} \cos \alpha_{m}+B_{z m a} \sin \alpha_{m}
$$

the angle derivative is simply

$$
\frac{\partial B_{m a}}{\partial \alpha_{m}}=-B_{r m a} \sin \alpha_{m}+B_{z m a} \cos \alpha_{m} .
$$


Note that this is also the expression for the poloidal magnetic field perpendicular to the probe axis and that any orientation misalignment can not be distinguished from a parasitic sensitivity of the probe winding in the normal direction.

Distribution of the current in the toroidal field coil connections. The connection between two adjacent toroidal field coils has been modelled by two separate coils with a given distribution of the total current in both coils (section 2.1). This distribution is quite difficult to establish and a possible variation should be retained. If $\Delta \beta$ is this variation, the flux and field differences are respectively

$$
\left(M_{f T 1}-M_{f T 2}\right)(\Delta \beta) I_{T}
$$

and

$$
\left(B_{m T 1}-B_{m T 2}\right)(\Delta \beta) I_{T}
$$

where $I_{T}$ is the toroidal field current.

\subsection{Determination of the corrections}

To recover a good consistency level in the magnetic data and reach the required precision, all the small corrections listed before must be determined. Even if this represents a large number of parameters, 539, this is feasible since typically each signal must be corrected for 3 to 4 parameters but provides as mentioned 19 independent measurements, giving a total number of 2603 measurements.

The corrections in these parameters are determined by making the flux and magnetic field differences as small as possible. Scanning the possible sources of error, these differences are: 


$$
\begin{aligned}
& \Delta \psi_{f}=\psi_{f}-\left(M_{f a}+M_{f a} \cdot \Delta G_{a a}+\frac{\partial M_{f c}}{\partial R_{c}} \cdot \Delta R_{c c} \cdot T_{c a}\right. \\
& +\frac{\partial M_{f c}}{\partial Z_{c}} \cdot \Delta Z_{c c} \cdot T_{c a}+M_{f c} \cdot \frac{\partial T_{c a}}{\partial \beta} \cdot \Delta \beta+\Delta G_{f f} \cdot M_{f a} \\
& \left.+\Delta R_{f f} \cdot \frac{\partial M_{f a}}{\partial R_{f}}+\Delta Z_{f f} \cdot \frac{\partial M_{f a}}{\partial Z_{f}}\right) \cdot I_{a}
\end{aligned}
$$

and

$$
\begin{aligned}
& \Delta B_{m}=B_{m}-\left(B_{m a}+B_{m a} \cdot \Delta G_{a a}\right. \\
& +\frac{\partial B_{m} c}{\partial R_{c}} \cdot \Delta R_{c c} \cdot T_{c a}+\frac{\partial B_{m} c}{\partial Z_{c}} \cdot \Delta Z_{c c} \cdot T_{c a} \\
& +B_{m c} \cdot \frac{\partial T_{c a}}{\partial \beta} \cdot \Delta \beta+\Delta G_{m m} \cdot B_{m a}+\Delta R_{m m} \cdot \frac{\partial B_{m a}}{\partial R_{m}} \\
& \left.+\Delta Z_{m m} \cdot \frac{\partial B_{m a}}{\partial Z_{m}}+\Delta \alpha_{m m} \cdot \frac{\partial B_{m} a}{\partial \alpha_{m}}\right) \cdot I_{a}
\end{aligned}
$$

Matrix notation has been used in this set of equations and twin indexing symbolises a diagonal matrix. $\psi_{f}$ and $B_{m}$ represent the flux and the magnetic field as they are measured. The correction parameters are determined by minimising a cost function combining the residual errors:

$$
\chi^{2}=\sum_{f, k} w_{F f}^{2} \Delta \psi_{f k}^{2}+\sum_{m, k} w_{B m}^{2} \Delta B_{m k}^{2}
$$

where $k$ is an index running over the data points and $w_{F f}$ and $w_{B m}$ are weights, set to the inverse of the experimental error, typically $1 \mathrm{mWb}$ and $0.5 \mathrm{mT}$. Note that for this equation set to give a unique solution, the vertical position of one element and the absolute gain of one measurement must be fixed: $\Delta Z_{c}=0$ for coil $\mathrm{A}$ and $\Delta G_{f}=0$ for loop number 1 have been chosen. The resulting corrections are shown in figure 15 . They are remarkably small. This means that event if no signal had been calibrated except one and that no coil and probe position were known, it would still be possible to get a meas- 
urement precision within a few percent and to place the coils and the sensors within a few $\mathrm{mm}$. This is not true for the toroidal field coil connections, whose field, by its dipolar nature, is 500 times smaller than the other induced field and escapes the experimental precision. The improvement brought by these corrections can be quantified by the root mean square of the residual errors in the flux and magnetic field: these drop from $4.6 \mathrm{mWb}$ and $2.1 \mathrm{mT}$ to $0.5 \mathrm{mWb}$ and $0.3 \mathrm{mT}$, which is clearly visible in figures 13 and 14 .
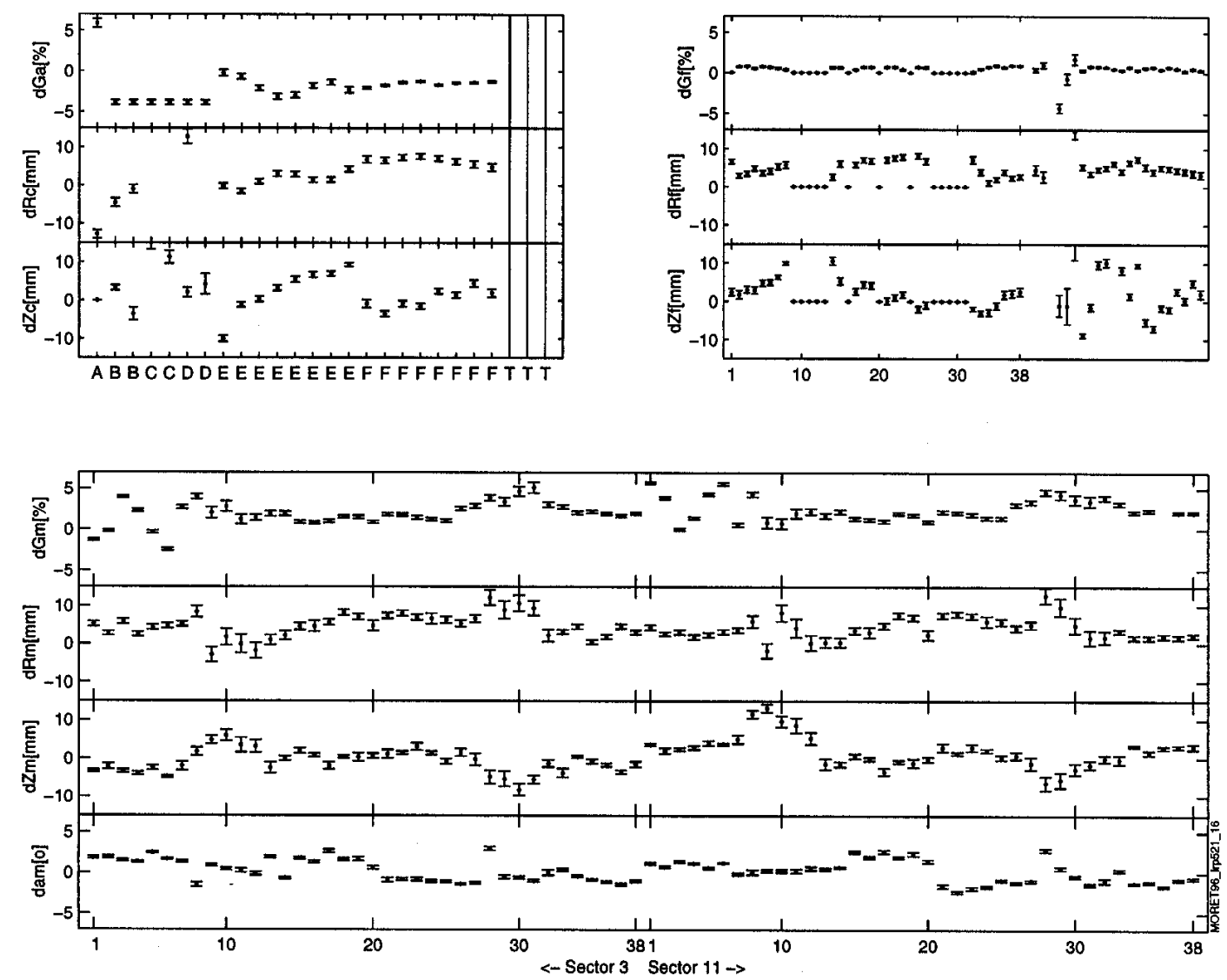

Fig. 15 Value of the corrections in the coil current calibration and on their location, on the flux loop calibration and their location and on the magnetic probe calibration, location and orientation. These corrections were derived without imposing any constraint on their amplitude.

The magnitude of the corrections however exceeds expected values. Moreover some corrections are statistically correlated and in that sense, a correct determination is much more difficult. This effect can be quantified by the covariance matrix of the corrections computed when minimising the cost function, plotted in figure 16. For example there is a dominant and obvious corre- 
lation between the signal calibrations: any decrease in the coil currents implies a positive compensation in the flux and field measurements.

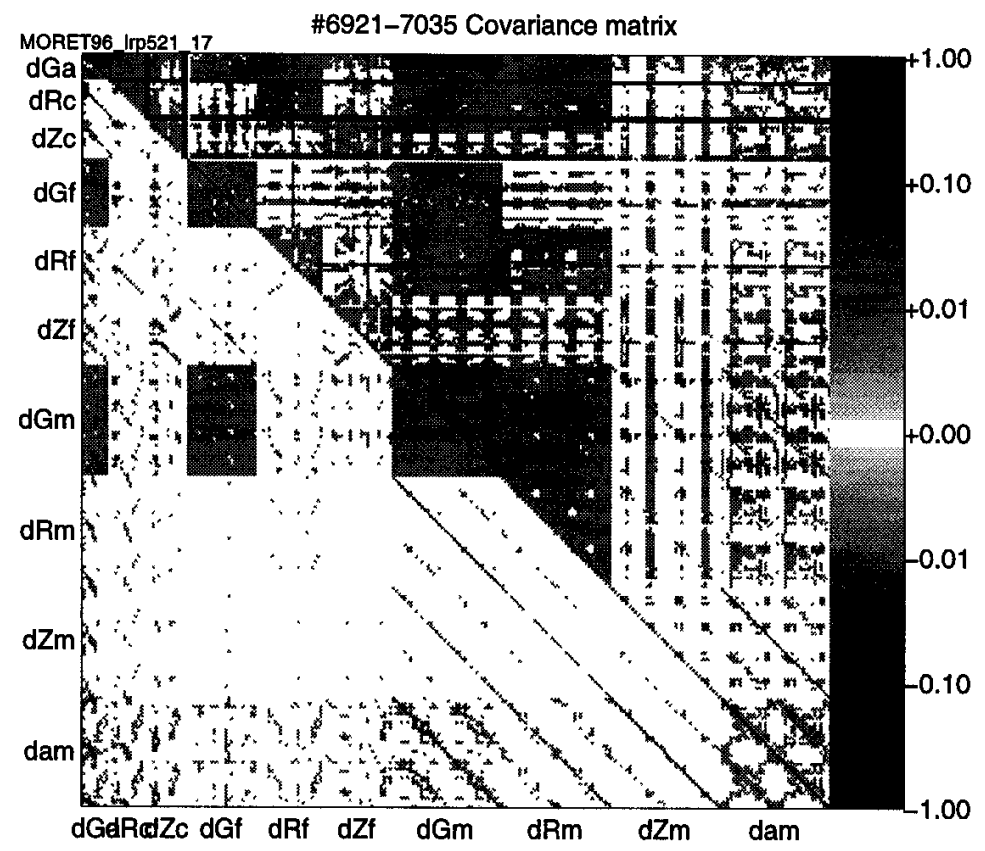

Fig. 16 Colour plot of the covariance matrix between the corrections. Upper half matrix represents the free correction fitting, lower half matrix is for constraint correction fitting.

Reduction of both the correction amplitude and their correlation may be obtained by explicitly imposing small corrections. This is done with a new cost function that includes a contribution for non zero corrections:

$$
\begin{aligned}
& \chi^{2}=\sum_{f, k} w_{F f}^{2} \Delta \psi_{f k}^{2}+\sum_{m, k} w_{B m}^{2} \Delta B_{m k}{ }^{2}+\sum_{a} w_{G a}^{2} \Delta G_{a}^{2} \\
& +\sum_{c} w_{R c}^{2} \Delta R_{c}^{2}+\sum_{c} w_{Z c}^{2} \Delta Z_{c}^{2}+w_{\beta}^{2} \Delta \beta+\sum_{f} w_{G f}^{2} \Delta G_{f}^{2} \\
& +\sum_{f} w_{R f}^{2} \Delta R_{f}^{2}+\sum_{f} w_{Z f}^{2} \Delta Z_{f}^{2}+\sum_{m} w_{G m}^{2} \Delta G_{m}^{2} \\
& +\sum_{m} w_{R m}^{2} \Delta R_{m}^{2}+\sum_{m} w_{Z m}^{2} \Delta Z_{m}^{2}+\sum_{m} w_{\alpha m}^{2} \Delta \alpha_{m}^{2}
\end{aligned}
$$

The new weights are chosen to be the inverse of the expected error in the corresponding parameters, as listed before and in Table IV. All deduced corrections, shown in figure 17 , are now limited to a tolerable amplitude. This new cost function of course implies an increase in the residual errors of the flux and field measurements, but the appropriate choice in the weights limits these 
residues to respectively $0.8 \mathrm{mWb}$ and $0.4 \mathrm{mT}$. This represents only a slight increase compared to the free parameter case and corresponds to measurement precision much better than expected. This can be visualised in figure 13 and 15 where for both the flux and the field measurements, the experimental differences and the individual contribution of each correction to the overall difference have been plotted. The improvement in the parameter decorrelation is significant as can be seen in figure 16.

\begin{tabular}{|c|c|}
\hline$w_{F f}$ & $1 \mathrm{mWb}$ \\
\hline$w_{B m}$ & $1 \mathrm{mT}$ \\
\hline$w_{G a}$ & $1 \%$ \\
\hline$w_{R c}, w_{Z c}$ & $5 \mathrm{~mm}$ \\
\hline$w_{\beta}$ & $10 \%$ \\
\hline$w_{G f}$ & $0.5 \%$ \\
\hline$w_{R f}, w_{Z f}$ & $2 \mathrm{~mm}$ \\
\hline$w_{G m}$ & $1 \%$ \\
\hline$w_{R m}, w_{Z m}$ & $2 \mathrm{~mm}$ \\
\hline$w_{\alpha}$ & $1.8^{\circ}$ \\
\hline
\end{tabular}

Table IV. Inverse weights used in determining the corrections. 

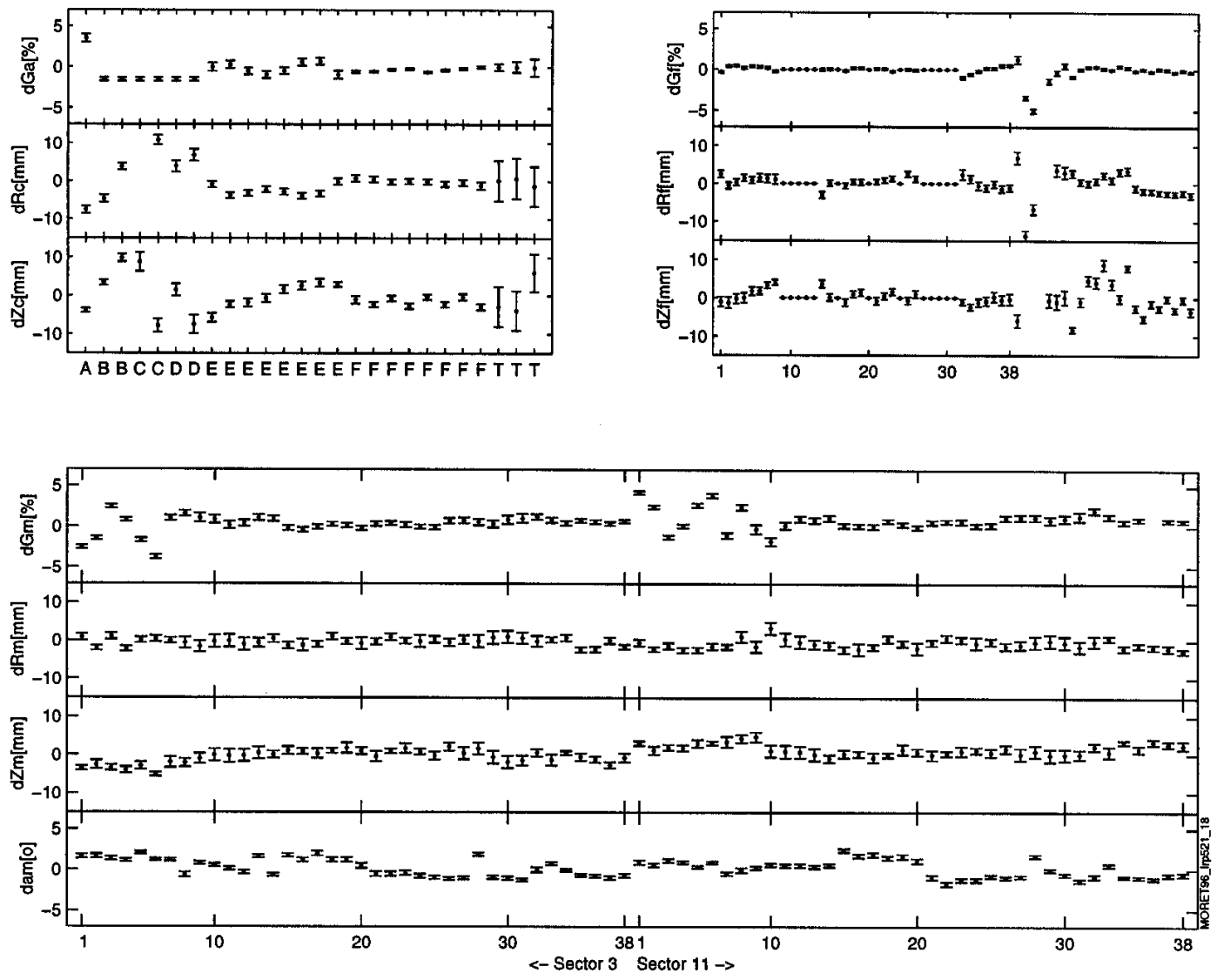

Fig. 17 Same as figure 15 but with a modified cost function that imposes the corrections to remain small.

It is important to note at this stage that this covariance matrix does not depend on the measurements, except for a scaling factor. It depends only on the geometry of the coils and the sensors and on the expected calibration errors. Thus the improvements that can be expected from such a coherence analysis on a future device can already be calculated during its design phase. This exercise would give useful information on the ability of the planned sensor set to reach a desired precision.

\subsection{Linearity}

It could be imagined that the corrections to be applied to the various calibration arise from a non-linearity in the sensors or electronics. In this case the corrections are not valid for the entire measurement range. To check this the same exercise has been repeated with all currents in the coil divided by two. Both correction sets are plotted on the same graphic in figure 18. Within 
the error bars there is no difference in most of the derived corrections, confirming the good linearity of the system. This however is not strictly true for some current measurements and that reinforces the fear that the current sensors which are based on a ferromagnetic circuit are not adequate when highly linear measurements are required.
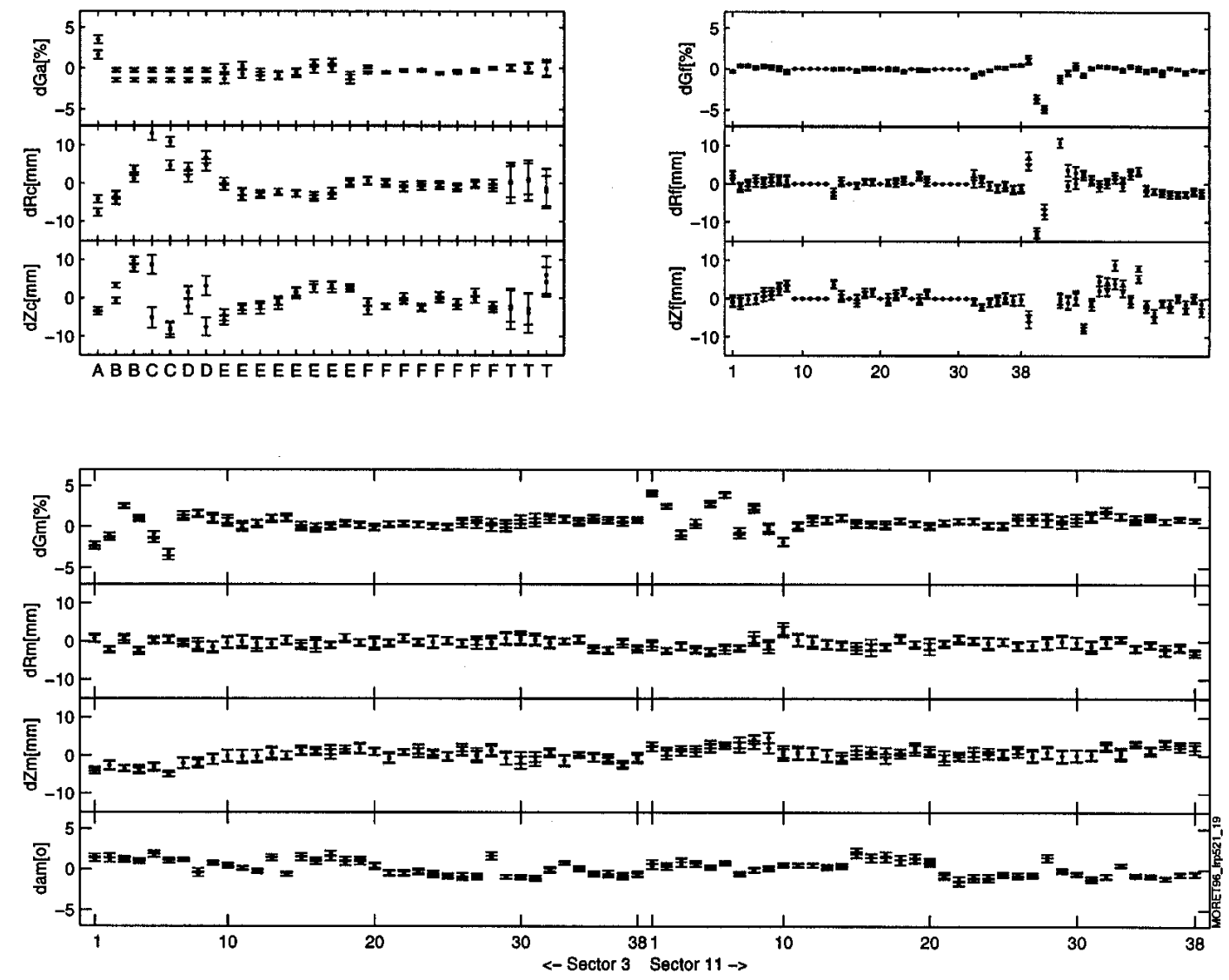

Fig. 18 Superposition of the corrections derived with two coil current amplitudes.

\subsection{Partial flux loops}

All flux loops that bypass ports have been excluded from the above exercise, because these detours would obviously impair an exact determination of the loop location. The data recorded for these loops can however be used to experimentally derive the coefficients used to combine them into an ideal flux loop set (section 4.2), simply by searching for those coefficients that give the combination of the ideal loop fluxes as close as possible to the measured fluxes for all data points. The resulting coefficients, shown in figure 19, are not far 
from the crude calculation based on the constructional dimensions but are certainly more precise.
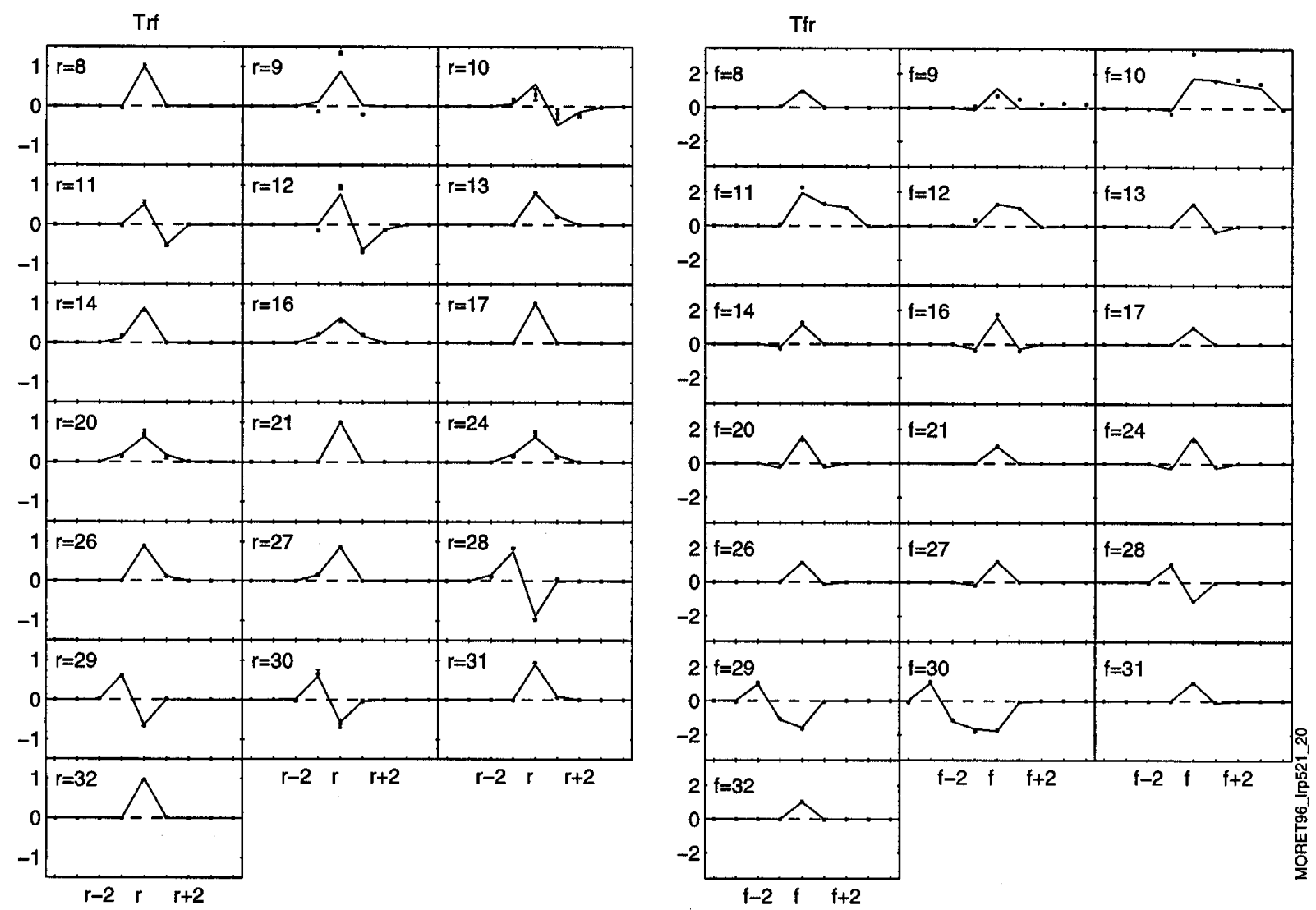

Fig. 19 Transformations from the relative flux loop measurement to the corresponding ideal fluxes. For each flux loop, the coefficient for that loop is centrally plotted together with those of its neighbour. The coefficients derived from constructional dimensions are plotted as solid lines while those derived from measurements are represented by dots.

\subsection{Toroidal field compensation}

As mentioned before, the connections between the toroidal field coils and their return loop produce a poloidal magnetic field. The associated flux is properly detected by those flux loops that make a complete turn (figure 20). For those loops that bypass ports, either their complicate path and the fact that they may not average the toroidal field ripple to zero certainly produce a parasitic sensitivity to the toroidal magnetic field itself that must be compensated. 
The picture is less clear for the magnetic field measurements because the parasitic sensitivity to the toroidal field is much larger than the associated poloidal field. This is due to misalignment of the probe windings and to the separation in the vacuum feedthroughs of the otherwise twisted pair cable. This however remains a small fraction of the effective field, about $1 \%$.
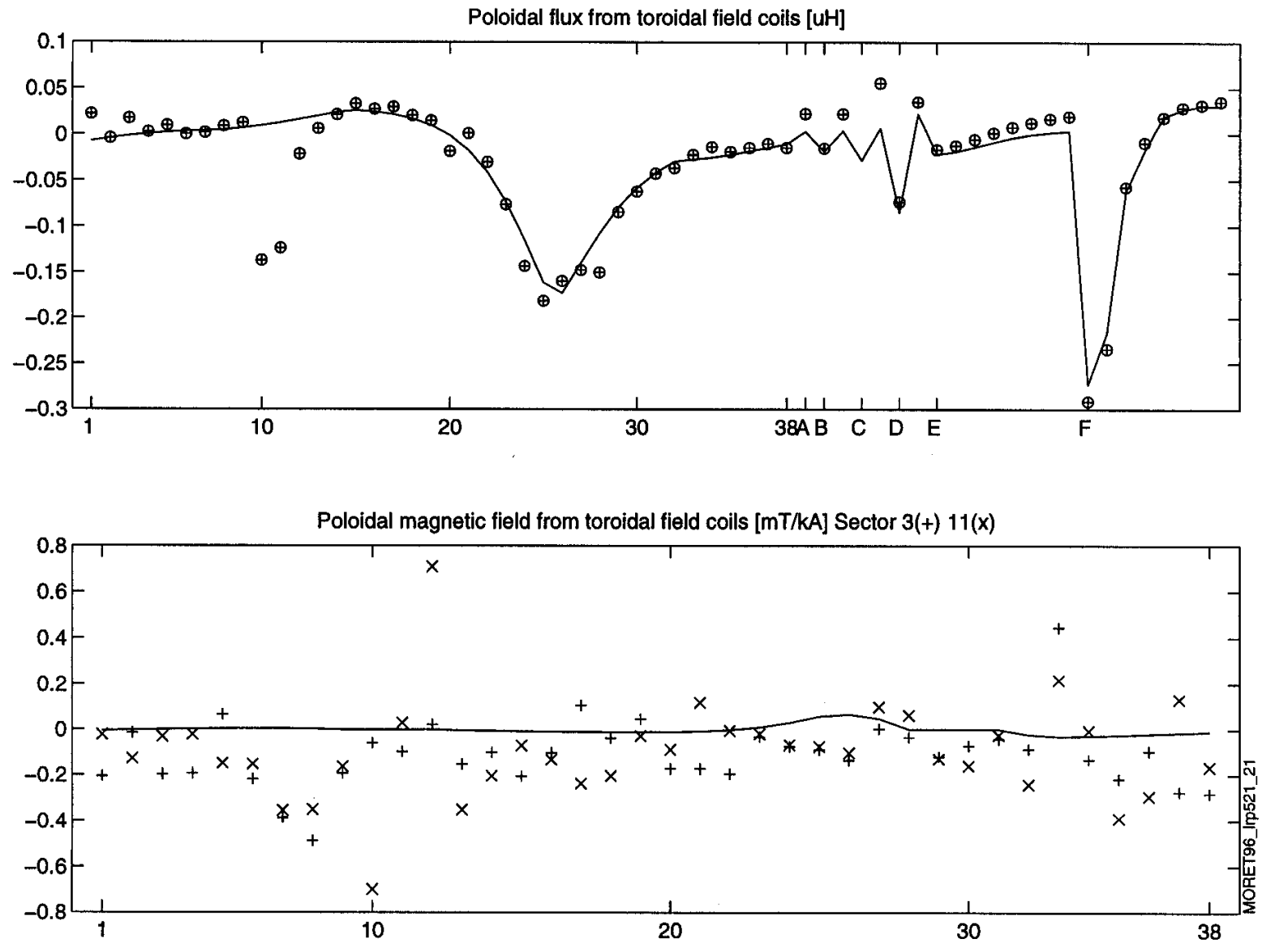

Fig. 20 Poloidal flux and poloidal magnetic field produced by the toroidal field coil connections at the position of the loops and probes. The solid line represents the values derived from constructional dimensions while crosses and circles are the measured values.

\subsection{Magnetic triangulation}

The original idea for studying the coherence of all the magnetic measurements was to verify the location of loops and probes using the fact that, as seen in figure 21 , the iso-flux surfaces for all the coils cross themselves with very different angles at each loop location. Thus the determination of the exact location of the sensors by magnetic triangulation was thought to be possible. A 
zoom in the crossing area (figure 21) shows how a geometric resolution of the problem would be difficult. The formal approach used proved itself much more suited. In addition it allows to introduce corrections other than geometrical effects, such as calibration errors. But just for the fun to see it, the corrected flux surfaces are also plotted in figure 21 to show to which extend the crossing area has been shrunk.
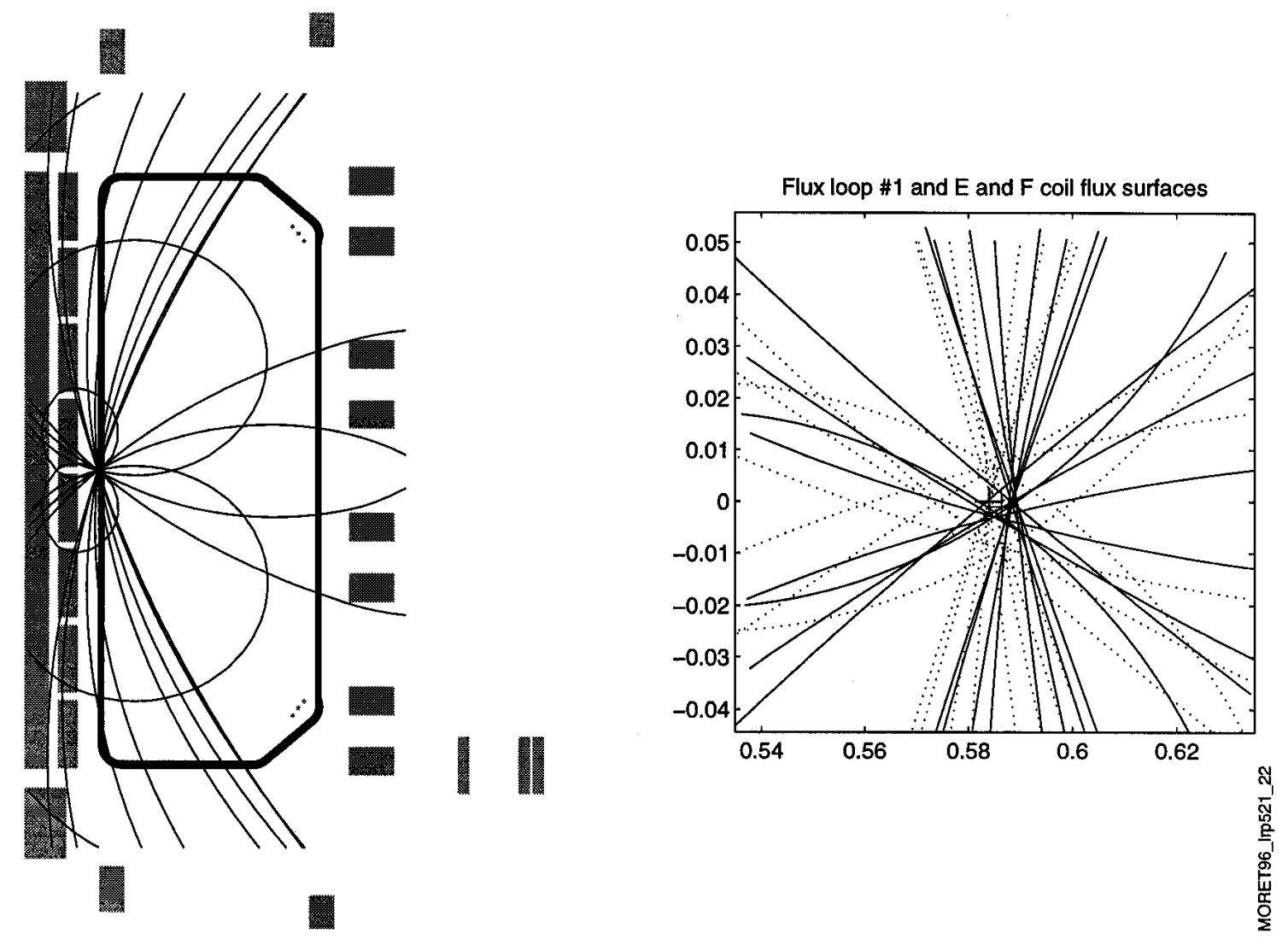

Fig. 21 Magnetic triangulation. Contours of constant flux at the flux level measured by loop 1 due to each $E$ and $F$ coil currents. On the enlarged plot these contours and the loop location are shown before applying the corrections (dotted lines) and with the corrections (solid lines).

\section{Plasma current estimator}

Since no continuous Rogowsky coil has been installed around the vacuum vessel, the total plasma current must be estimated with an appropriate combination of the poloidal magnetic field probes. The disadvantage of such a discrete measurement is that it may not be precise and is certainly to some 
extent sensitive to currents flowing outside the integration path, especially the coil currents and vessel eddy currents.

A first simple choice for the linear combination coefficients can be based on a polygonal integration contour given by the probe locations and a trapeze approximation of the integral. The error in the estimated total enclosed current mainly comes from currents localised near this contour, as can be seen in figure 22. In practice however, no significant plasma current density is to be found in this region and the total plasma current estimator is excellent.

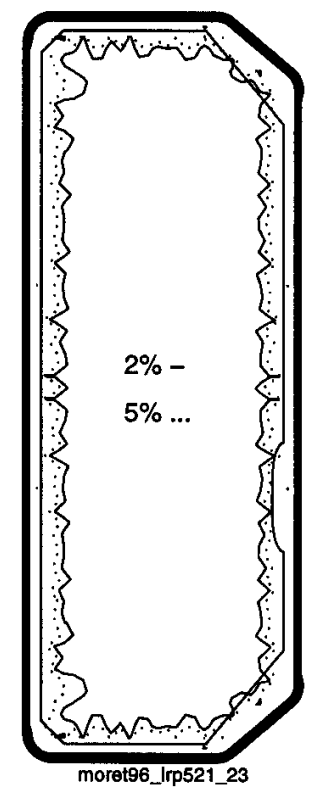

Fig. 22 Contours of the error on the discretised plasma current estimator for currents inside the integration path.

Sensitivity to coil currents is substantial, especially to those coils near vertices of the polygonal integration contour, namely F1, F2, F7 and F8, for which it reaches $0.8 \mathrm{~A} / \mathrm{A}$. This effect is however easily measured and compensated. The experimentally deduced compensation coefficients together with their constructional estimate are given in figure 23. 


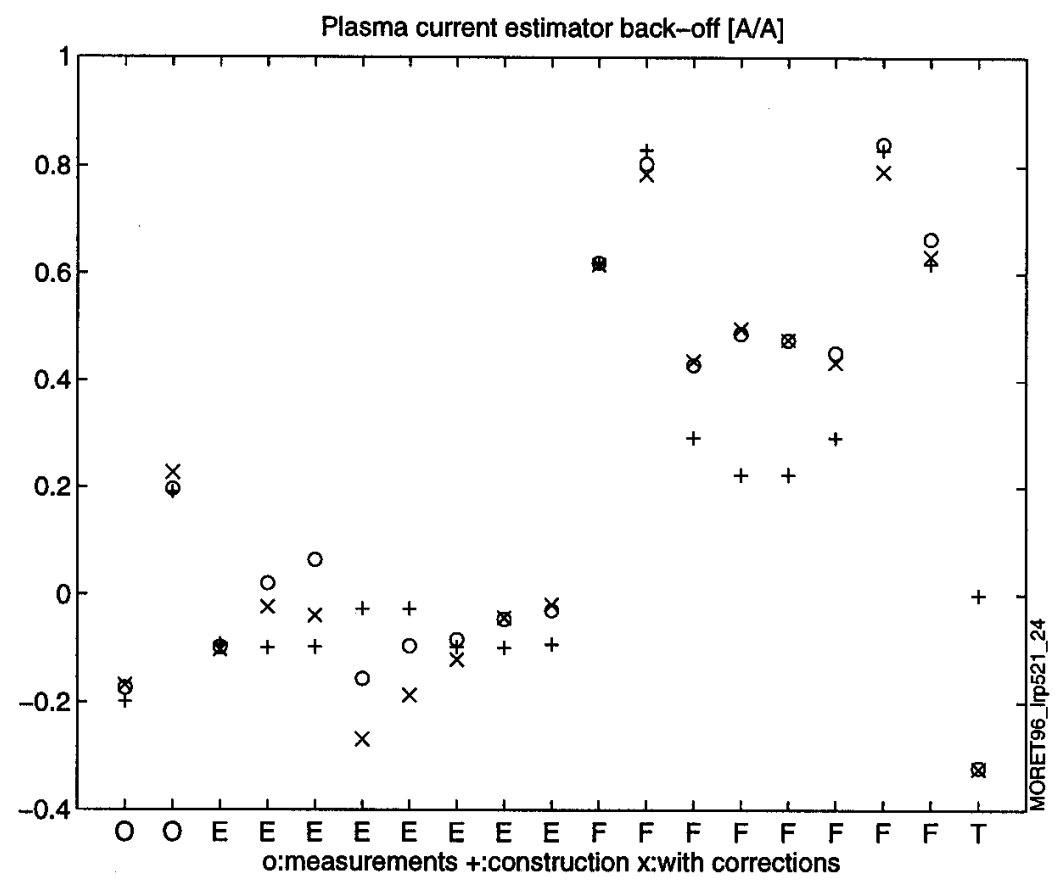

Fig. 23 Contribution to the discretised plasma current estimator of the coil currents: derived from constructional dimensions (+), including calibration and location corrections $(x)$, measurements $(\bullet)$.

The influence of the eddy currents in the vessel to the trapezoidal plasma current estimator has been modelled for each up-down symmetric vessel eigenmode. As it can be noticed on figure 24, this influence is small for low order eigenmodes, but it peaks around mode numbers 38, 76 and 114. The current distribution of these eigenmodes coincides with the probe array discretisation, giving a resonant contribution. Fortunately these highly spatially structured current distributions are difficult to couple to and their excitation amplitude remains small and does not significantly perturb the plasma current estimation. 


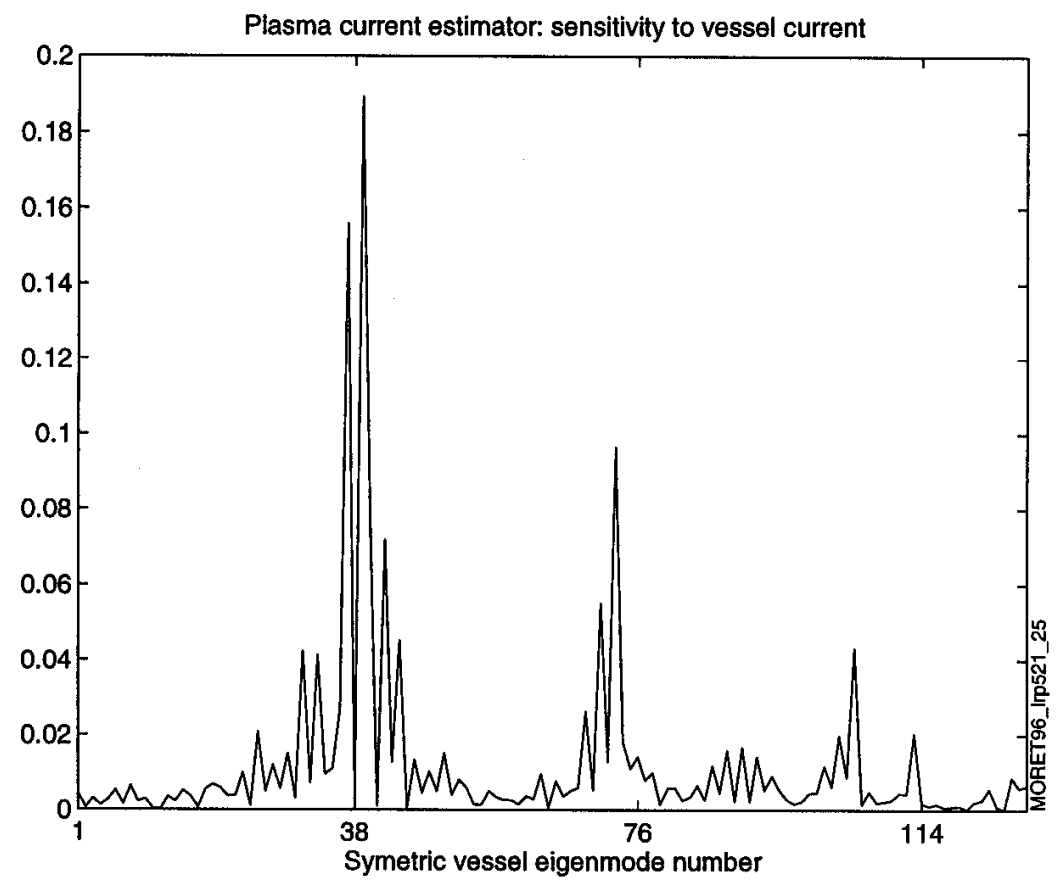

Fig. 24 Sensitivity of the discretised plasma current estimator to the symetric vessel eigenmodes.

\section{Conclusion}

The most useful conclusion for this presentation of the TCV magnetic measurements is certainly an criticism of the whole system, the positive features that would be repeated if TCV had to be rebuilt and the negative points that cause minor or major difficulties and that would or will have to be improved. The most salient points are listed here, categorised by $\boldsymbol{v}$ and $x$ symbols:

$\checkmark$ The number and location of both the flux loops and the magnetic probes has been found to be adequate even though the extreme flexibility of TCV poses severe requirements on the system. Up to date, no difficulties have been encountered that would be simply resolved by an expanded set of magnetic measurements.

$x$ This positive statement is soured to some extent by the partial flux loops on the top and bottom of the vessel. Of course detours around the ports are unavoidable, but the partial flux loops exacerbate the difficulties. In order to estimate the flux in the region covered by these loops, it is necessary to 
interpret exactly the signal of each partial loop and to sum them up to the point of interest, thus accumulating errors. Full turn loops even with large detours around the biggest ports would much less suffer from this problem, only in a proportion given by the ratio of the detour area to the loop separation. The estimation of the flux in these regions could also be improved by measurement at some positions of the normal (vertical) magnetic field.

$\checkmark$ Flux loops wound directly on the coils are not necessary either for real time control or for equilibrium reconstruction. Fast variation of the flux near the coils makes the measurement very sensitive to the exact loop position and to the conductor distribution within the coils.

$x$ The specifications on the precision of the coil current sensors were not sufficiently severe. The consequences of this lack of precision and linearity are problems when using this data, for example, in the reconstruction of the equilibrium and of the magnetic field topology during breakdown. In both cases this uncertainty has been to some extent balanced by the large redundancy in the whole magnetic data set.

- Major problems have been encountered with the somewhat fragile wire used to wind the magnetic probes. Firstly, the electrical isolation of the wire is guaranteed to only $250 \mathrm{~V}$ and as previously mentioned, very fast MHD events can give rise to voltage spikes exceeding $300 \mathrm{~V}$. Secondly, the mechanical strength of this wire is incompatible with the severe constraints of a tokamak environment. Wires have been broken during the installation process and short circuits have appeared between the central conductor and the shield, which are attributed to crushed wire in the bindings. To correct these problems, more robust replacement probes with a $1 \mathrm{~mm}$ diameter wire and a reduced area are under construction.

$x$ The magnetic probes have only been calibrated along their axis. This calibration should be extended to three spatial directions. This is a minor point that does not compromise the measurements, but the consistency analysis and the associated determination of the diagnostic quality would be easier if the parasitic sensitivity to a transverse field was known.

$\checkmark$ The precision and the reliability of the amplifying electronics have proved 
to be excellent in all respects. The absolute precision associated with an extensive and low error calibration, the linearity or the offset and drift level are largely adequate. No systematic failure has yet appeared that would jeopardise the use of the system, except a first version of the preamplifier that was not equipped with the high frequency signal attenuation and whose input stage was sometimes destroyed by the mentioned high voltage spikes.

$\checkmark$ A high level of consistency and a high precision in the magnetic data set has been reached, typically $1 \mathrm{mWb}$ and $0.5 \mathrm{mT}$ for the flux and magnetic field measurements respectively. Although a preliminary analysis indicated higher errors than expected, it has been possible to reduce these errors to an acceptable level by determining small corrections in the system calibration and in the coil, flux loop and magnetic probe locations, compatible with the known parameters of the system. The formal analysis method developed for this purpose yields a very precise knowledge of the magnetic measurement characteristics and a high degree of confidence in the data that this diagnostic produces.

\section{Appendix 1: Sign convention (figure 25)}

The cylindrical coordinate system $(R, \varphi, Z)$ is chosen as follows: the $Z$ axis points towards the top, the $Z=0$ plane coincides with the equatorial mid-plane; to keep a right hand coordinate system, the toroidal angle $\varphi$ is measured in the trigonometric positive direction seen from the top.

Integration contours of the flux loops run along the positive direction. Thus a positive flux is produced by a vertical field pointing towards the top and a radial field pointing towards the outside leads to a positive flux difference between a given flux loop and its neighbour located above.

A positive plasma current produces along the vacuum vessel a tangential poloidal field that is defined positive. 


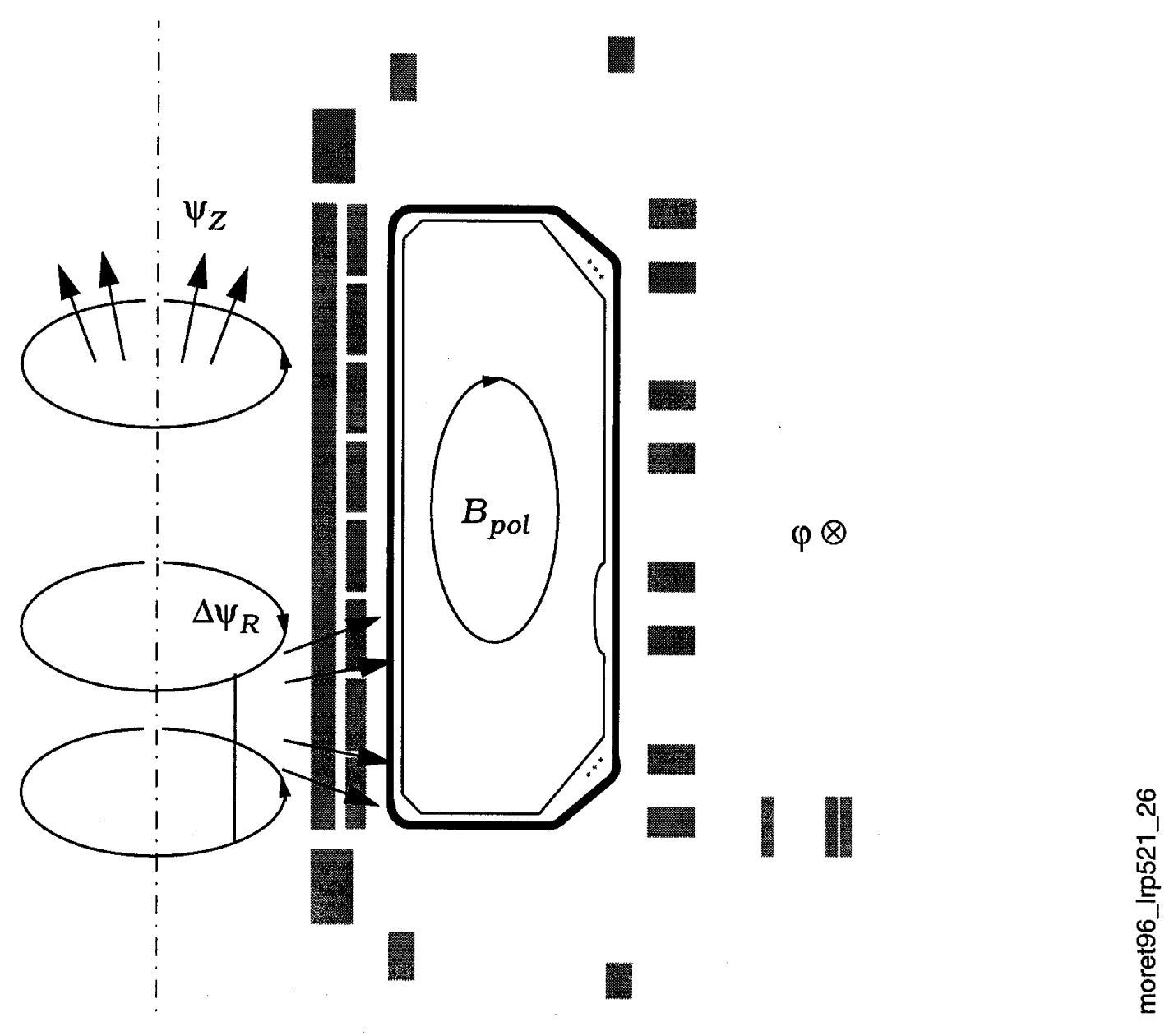

Fig. 25 Magnetic measurement sign convention.

Acknowledgement. We thank the entire TCV team for their effort. This work was partly supported by the Fonds National Suisse de la Recherche Scientifique.

\section{References}

[1] F. Hofmann, J.B. Lister, M. Anton et al., Plasma Phys. Contr. Fusion 36 (1994) B277.

[2] F. Hofmann, S.C Jardin, F.B. Marcus et al., Proc. 14th Symp. on Fusion Technology, Avignon (1986) 687.

[3] J.-M. Moret, Fitting of transfer functions to frequency response measurements, CRPP Laboratory Report LRP 498/94, Lausanne, (1994).

[4] G. Flor, G. Manduchi, T.W. Fredian et al., Proc. 16th Symp. on Fusion Technology, London (1990) 1272. 